SPhT T-01/027

DFF $371 / 01 / 2001$

GE-TH-03/2001

hep-th/0103237

\title{
Exact Consequences of the Trace Anomaly in Four Dimensions
}

\author{
Andrea CAPPELLI \\ I.N.F.N. and Dipartimento di Fisica, \\ Largo E. Fermi 2, I-50125 Firenze, Italy \\ e-mail: andrea.cappelli@fi.infn.it \\ Riccardo GUIDA \\ CEA-Saclay, Service de Physique Théorique, \\ F-91191 Gif-sur-Yvette, France \\ e-mail: guida@spht.saclay.cea.fr \\ Nicodemo MAGNOLI \\ I.N.F.N. and Dipartimento di Fisica, \\ Via Dodecaneso 33, I-16146 Genova, Italy \\ e-mail:magnoli@ge.infn.it.
}

\begin{abstract}
The general form of the stress-tensor three-point function in four dimensions is obtained by solving the Ward identities for the diffeomorphism and Weyl symmetries. Several properties of this correlator are discussed, such as the renormalization and scheme independence and the analogies with the anomalous chiral triangle. At the critical point, the coefficients $a$ and $c$ of the four-dimensional trace anomaly are related to two finite, scheme-independent amplitudes of the three-point function. Offcriticality, the imaginary parts of these amplitudes satisfy sum rules which express the total renormalization-group flow of $a$ and $c$ between pairs of critical points. Although these sum rules are similar to that satisfied by the two-dimensional central charge, the monotonicity of the flow, i.e. the four-dimensional analogue of the $c$-theorem, remains to be proven.
\end{abstract}

February 2001 


\section{Introduction}

The Zamolodchikov $c$-theorem [1] states that the two-dimensional unitary field theories are characterized by a positive function of the coupling constants $c(g)$ which decreases along the renormalization-group flow; moreover, its value at the fixed points is equal to the trace-anomaly number $c$. Several works have suggested that the theorem could extend to four dimensions 7 : at criticality, the candidate $c$-function would again reduce to the coefficient of the the Euler density in the trace anomaly [3]. This conjecture is supported by many examples of non-trivial flows in supersymmetric gauge theories, which have been analysed via the Seiberg-Witten electric-magnetic duality [6] and the AdS supergravitygauge theory correspondence [7] [8]. Two proofs of the four-dimensional theorem have also been proposed, although one is rather controversial [9], and the other one relies on a strong dynamical hypothesis [10].

These results and conjectures motivate further analyses of the physical consequences of the four-dimensional trace anomaly

$$
g^{\mu \nu}\left\langle T_{\mu \nu}\right\rangle \equiv\langle\Theta\rangle=\lambda\left(a E-3 c W+a^{\prime} D^{2} \mathcal{R}+r \mathcal{R}^{2}\right), \quad \lambda \equiv \frac{1}{2880 \cdot 4 \pi^{2}} .
$$

In this equation, $E$ denotes the Euler density $\left(\chi \propto \int \sqrt{g} E\right.$ is the Euler characteristic), $W$ the square of the Weyl tensor and $\mathcal{R}$ the curvature scalar (see Appendix A for our conventions). Some properties of the trace anomaly (1.1) are known since a long time [12] [13]: the fact that the Wess-Zumino consistency conditions imply $r=0$ [14] 15] and that the coefficient $a^{\prime}$ is scheme dependent, because it can be changed by adding a local counterterm to the effective action [13]. An interesting study of the four-dimensional trace anomaly (and its higher-dimensional analogues) has been done in Ref. [16], by deriving the corresponding terms in the regularized effective action within the framework of the epsilon-expansion. It was found that the topological invariant Euler term corresponds to an effective action which is finite and non-local as $\epsilon \rightarrow 0$; this is the same feature of the chiral anomaly [12]. On the other hand, the $W$ term in (1.1) corresponds to a singular effective action with finite scale variation.

In this work, we relate the four-dimensional trace anomaly to some physical quantities: i) we express the universal parameters $a$ and $c$ in terms of finite scheme-independent correlation functions at criticality; ii) away from criticality, we provide exact sum rules for the renormalization-group flow of $a$ and $c$.

* For a short review, see Ref. 2]; for early attempts, see the Refs. [3] [4] [5].

$\dagger$ Note that the normalization of the four-dimensional stress tensor in Eq.(1.1) differs from that in Refs. 51 111 by the factor $1 / 4 \pi^{2}$; nevertheless, the values of the anomaly coefficients for the free conformalinvariant scalar field are still normalized to $a=c=1$.

$\ddagger$ These flows have also been analysed by the Refs. 4 [10 within the perturbative epsilon-expansion. 
The analogous two-dimensional results are well known [17]: the coefficient $c$ of the trace anomaly $\langle\Theta\rangle=-c \mathcal{R} / 12$ is unambiguously defined by the stress-tensor two-point function $\langle T(z) T(0)\rangle=c / 2 z^{4}$ at criticality, which is a finite and well-defined correlator. Furthermore, the renormalization-group flow of $c$ is constrained by the Cardy sum rule, which involves the off-critical correlator [18]:

$$
c_{U V}-c_{I R}=\frac{3}{4 \pi} \int_{|x|>\epsilon} d^{2} x x^{2}\langle\Theta(x) \Theta(0)\rangle=\int_{\epsilon}^{\infty} d s c(s)>0 .
$$

In the above Equation, $c_{U V}$ and $c_{I R}$ are the central charges of, respectively, the ultraviolet and infrared fixed points connected by the renormalization-group trajectory, and the $x$ integral is evaluated at any point along the trajectory. Equation (1.2) also shows the form of the sum rule in momentum space [5]: it involves the positive-definite spectral measure $c(s) d s$, which is obtained from the imaginary part of $\langle\Theta(p) \Theta(-p)\rangle$ at momentum $p^{2}=s$. In four dimensions, the relation of the trace anomaly (1.1) to finite correlators requires the study of the three-point function in flat spaces or of the two-point function in curved space; their general expressions at criticality (in coordinate space) have been found in the Refs. 19] 20] and [21], respectively.

In this paper, we obtain the general form of the three-point function in (flat) momentum space at and off criticality: we write it as a sum of scalar amplitudes (form factors) times tensor structures, which solve the Ward identities for the diffeomorphism and Weyl symmetries (the latter being imposed at criticality). In addition, we write dispersion relations for the renormalized amplitudes following closely the well-known analysis of the chiral triangle [22].

At criticality, we can identify the three-point amplitudes which correspond to the terms in the trace anomaly, disentangling the scheme-dependent amplitude related to $a^{\prime}$ in (1.1) from the scheme-independent ones corresponding to the coefficients $a$ and $c$. Any amplitude can be singled out from the general expansion of $\langle T T T\rangle$ by solving a linear system: we obtain some reduction formulae which can be useful for computing $a$ and $c$ in interacting theories; these relations could also suggest further physical consequences of the trace anomaly.

Away from criticality, the imaginary parts of the $a$ and $c$ amplitudes satisfy two exact sum rules, which express the total variation of $a$ and $c$ under the renormalization-group flow between two fixed points; these sum rules follow from the analysis of the general structure of $\langle T T T\rangle$ and are analogous of the two-dimensional result (1.2).

In two dimensions, the imaginary part of the two-point function $c(s)$ is positive definite by unitarity; the sum rule (1.2) then implies that $c$ decreases along the renormalization-

\footnotetext{
$\S$ Hereafter, we shall mostly use the Euclidean signature (see Appendix A for our conventions).
} 
group flow. In four dimensions, the property $a_{U V}>a_{I R}$ could be similarly proved if the imaginary part of the $a$ amplitude were positive definite. In general, we do not expect that three-point functions, related to decay amplitudes, should have a definite sign; nevertheless, we should mention the positivity condition proposed in Ref. [20], coming from the classical weak-energy condition [23], which implies the positivity of the $a$ coefficient at criticality. The consequences of this condition on the three-point amplitudes off-criticality remain to be analysed.

The plan of the paper is the following: in Section 2, we briefly recall the dispersive analysis of the chiral triangle $\langle A V V\rangle$ of Ref. 22]; this lets us outline some features which arise for $\langle T T T\rangle$ too. In this Section, we also give the definition of the stress-tensor operator and repeat the dispersive analysis of $\langle T T\rangle$ [5] 24]. Appendix $\mathrm{A}$ and B contain our notations for the curved-space calculus and the derivation of the Ward identities, respectively.

In Section 3, we present the analysis of $\langle T T T\rangle:$ i) the decomposition in scalar amplitudes times the tensors solving the diffeomorphism Ward identity (the tensor basis is constructed in Appendix Q); ii) the conditions on the amplitudes which are imposed by the (anomalous) Weyl symmetry at criticality. Owing to the large number of possible tensor structures, we make an extensive use of algebraic programs (longer formulas are given in Appendix D and the full output is available as a Mathematica [26] notebook). In this Section, we also discuss the Ward identities that follow from flat-space conformal invariance at criticality.

In Section 4, we show how to extract the scalar amplitudes in the $\langle T T T\rangle$ expansion from an explicit expressions of this correlator; in particular, we obtain the reduction formulae which determine the trace anomaly coefficients $a$ and $c$ at criticality. Next, we discuss the renormalization-group flow of the corresponding amplitudes off-criticality and derive the sum rules analogues to Eq.(1.2). In Section 5, we test the reduction formulas and the sum rules by computing the flows of the free massive scalar and fermion theories. In the Conclusions, we briefly discuss the possible attempts towards the $c$-theorem in four dimensions.

\section{Dispersive analysis of the chiral triangle and of the stress- tensor two-point function}




\subsection{Analysis of $\langle A V V\rangle$}

We start by recalling the well-known analysis of the three-point function of one axial and two vector currents [22]:

$$
\begin{aligned}
\Gamma_{\mu \alpha_{1} \alpha_{2}} & \equiv \int d^{4} x_{1} d^{4} x_{2} \mathrm{e}^{-i k_{1} x_{1}-i k_{2} x_{2}}\left\langle A_{\mu}(0) V_{\alpha_{1}}\left(x_{1}\right) V_{\alpha_{2}}\left(x_{2}\right)\right\rangle \\
q^{\mu} & =k_{1}^{\mu}+k_{2}^{\mu}, \quad k_{1}^{2}=k_{2}^{2} \equiv k^{2} .
\end{aligned}
$$

We shall discuss the renormalized correlator, i.e. we shall not explicitly introduce bare expressions and counterterms, although some finite scheme-dependent terms will occur. The space-time symmetries allow to decompose (2.1) in tensor structures times scalar amplitudes $A_{i}=A_{i}\left(q^{2}, k_{1}^{2}, k_{2}^{2}\right)$; we consider the symmetric point $k_{1}^{2}=k_{2}^{2}$, in order to fully exploit the Bose symmetry $\left(k_{1}^{\alpha_{1}} \leftrightarrow k_{2}^{\alpha_{2}}\right)$ of the correlator. The tensor notation can be simplified by contracting the Lorentz indices with general polarization vectors $\left\{v^{\mu}, e_{1}^{\mu}, e_{2}^{\mu}\right\}$, following Ref. 27]. We then write:

$$
\begin{aligned}
\Gamma\left(v, e_{1}, e_{2}\right) & \equiv v^{\mu} e_{1}^{\alpha_{1}} e_{2}^{\alpha_{2}} \Gamma_{\mu \alpha_{1} \alpha_{2}}=A_{1}\left[v e_{1} e_{2}\left(k_{1}-k_{2}\right)\right] \\
& +A_{3}\left(\left[v e_{1} k_{1} k_{2}\right]\left(e_{2} \cdot k_{1}\right)+\left[v e_{2} k_{2} k_{1}\right]\left(e_{1} \cdot k_{2}\right)\right) \\
& +A_{5}\left(\left[v e_{1} k_{1} k_{2}\right]\left(e_{2} \cdot k_{2}\right)+\left[v e_{2} k_{2} k_{1}\right]\left(e_{1} \cdot k_{1}\right)\right) \\
& +A_{7}\left[e_{1} e_{2} k_{1} k_{2}\right]\left(v \cdot\left(k_{1}+k_{2}\right)\right)
\end{aligned}
$$

where we denoted:

$$
a_{\mu} b^{\mu} \equiv(a . b), \quad \epsilon_{\mu \nu \rho \sigma} a^{\mu} b^{\nu} c^{\rho} d^{\sigma} \equiv[a b c d]
$$

The tensor structures in (2.2) are not linearly independent in four dimensions, due to the identity [27]:

$$
(a . f)[b c d e]+(b . f)[c d e a]+(c . f)[d e a b]+(d . f)[e a b c]+(e . f)[a b c d]=0,
$$

which holds for any six-plet of vectors $\{a, b, c, d, e, f\}$. In its Bose symmetrized form, this identity implies that one of the scalar amplitudes in the expansion (2.2) is redundant. Actually, $\Gamma$ is invariant under the shift of amplitudes by an arbitrary function $f$ :

$$
A_{1} \rightarrow A_{1}-\left(k^{2}+\left(k_{1} \cdot k_{2}\right)\right) f, \quad A_{i} \rightarrow A_{i}-f, \quad \text { for } i=3,5,7 .
$$

Therefore, we can eliminate one of the amplitudes in (2.2): we choose $A_{7}=0$ to match the notations of Ref.222.

We consider a general theory with massive fermions and write the Ward identities for the conservation of the vector and axial currents which enter in $\langle A V V\rangle$ (the axial current is 
supposed anomalous). These Ward identities can be expressed in our notation by choosing longitudinal polarizations in $\Gamma\left(v, e_{1}, e_{2}\right)$, for example $e_{1}=k_{1}$. We then write,

$$
\begin{aligned}
\Gamma\left(v, k_{1}, e_{2}\right) & =0, \\
\Gamma\left(k_{1}+k_{2}, e_{1}, e_{2}\right) & =2 m \Gamma_{5}\left(e_{1}, e_{2}\right)+\frac{C}{\pi}\left[e_{1} e_{2} k_{1} k_{2}\right] ;
\end{aligned}
$$

$\Gamma_{5}\left(e_{1}, e_{2}\right)$ is the Fourier transform of the corresponding triangle $\left\langle J_{5} V V\right\rangle$, involving the pseudo-scalar density, whose tensor structure is:

$$
\Gamma_{5}\left(e_{1}, e_{2}\right)=-A_{9}\left[e_{1} e_{2} k_{1} k_{2}\right] .
$$

The resulting conditions on the scalar amplitudes are:

$$
\begin{aligned}
& 2 A_{1}=q^{2} A_{3}+2 k^{2}\left(A_{5}-A_{3}\right) ; \\
& 2 A_{1}=-\frac{C}{\pi}+2 m A_{9} .
\end{aligned}
$$

The quantity $C$ on the right-hand side of (2.9) is the chiral anomaly: $C$ cannot be put to zero because $\Gamma$ would vanish altogether for $m=0$ at the point $q^{2}=k^{2}=0$, which is not physically acceptable. Furthermore, $C$ can be shown to be a constant as follows [22]. Consider the imaginary parts of all amplitudes w.r.t. both variables $q^{2}$ and $k^{2}$ : they are finite quantities describing physical scattering processes, that satisfy non-anomalous Ward identities,

$$
\begin{aligned}
2 \operatorname{Im} A_{1}\left(q^{2}, k^{2}\right) & =\left(q^{2}-2 k^{2}\right) \operatorname{Im} A_{3}\left(q^{2}, k^{2}\right)+2 k^{2} \operatorname{Im} A_{5}\left(q^{2}, k^{2}\right), \\
\operatorname{Im} A_{1}\left(q^{2}, k^{2}\right) & =m \operatorname{Im} A_{9}\left(q^{2}, k^{2}\right) .
\end{aligned}
$$

It follows that the anomalous term has vanishing imaginary parts:

$$
C\left(q^{2}+i \epsilon, k^{2}\right)-C\left(q^{2}-i \epsilon, k^{2}\right)=0=C\left(q^{2}, k^{2}+i \epsilon\right)-C\left(q^{2}, k^{2}-i \epsilon\right)
$$

thus, $C$ is a dimensionless polynomial of $q^{2} / m^{2}$ and $k^{2} / m^{2}$ with finite $m \rightarrow 0$ limit, i.e. it is a constant.

Next, we write the dispersion relations w.r.t. $q^{2}$ :

$$
\begin{aligned}
& A_{1}\left(q^{2}, k^{2}\right)=A_{1}^{U V}+\frac{1}{\pi} \int d s \frac{\operatorname{Im} A_{1}\left(s, k^{2}\right)}{s-q^{2}}, \\
& A_{i}\left(q^{2}, k^{2}\right)=\frac{1}{\pi} \int d s \frac{\operatorname{Im} A_{i}\left(s, k^{2}\right)}{s-q^{2}}, \quad i=3,5,9 .
\end{aligned}
$$

The integrals in Eq. 2.13,2.14 are convergent due to the asymptotic behaviors,

$$
A_{i}\left(q^{2}, k^{2}\right) \sim q^{\operatorname{dim} \mathrm{A}_{\mathrm{i}}}, \quad \text { for } q^{2} \rightarrow \infty,
$$


following from dimensional analysis (up to logarithms which are supposed absent for $A_{1}$ ): $\operatorname{dim} \mathrm{A}_{1}=0, \operatorname{dim} \mathrm{A}_{3}=\operatorname{dim} \mathrm{A}_{5}=-2$ and $\operatorname{dim} \mathrm{A}_{9}=-1 ;$ only the amplitude $A_{1}$ (superficially divergent at the bare level) requires the subtraction term $A_{1}^{U V}$ in (2.13). Combining Eqs. (2.10,2.11) and (2.14), we can derive the sum rule:

$$
\frac{1}{\pi} \int d s \operatorname{Im} A_{3}\left(s, k^{2}\right)+\left(q^{2}-2 k^{2}\right) A_{3}\left(q^{2}, k^{2}\right)+2 k^{2} A_{5}\left(q^{2}, k^{2}\right)=2 m A_{9}\left(q^{2}, k^{2}\right) .
$$

The comparison of this sum rule with the original Ward identities (2.8,2.9) determines $A_{1}^{U V}$ in (2.13) as the chiral anomaly $C$ and yields the following sum rule:

$$
A_{1}^{U V}=-\frac{C}{2 \pi}=-\frac{1}{2 \pi} \int d s \operatorname{Im} A_{3}\left(s, k^{2}\right)
$$

Let us further analyse the Eqs. (2.8, 2.9,2.17) for $k^{2}=m^{2}=0: A_{5}$ and $A_{9}$ drop out and we find the explicit solution,

$$
\begin{aligned}
& A_{3}\left(q^{2}, 0\right)=-\frac{C}{\pi} \frac{1}{q^{2}}, \quad \operatorname{Im} A_{3}\left(q^{2}, 0\right)=C \delta\left(q^{2}\right), \\
& A_{1}\left(q^{2}, 0\right)=-\frac{C}{2 \pi}=-\frac{1}{2 \pi} \int d s \operatorname{Im} A_{3}(s, 0) .
\end{aligned}
$$

This is famous result 22 that the anomaly appears as a delta term in the imaginary part of a finite amplitude $\left(A_{3}\right)$. We also notice that in the general case $k^{2} \neq 0, m \neq 0$, the constancy of the sum rule (2.17) implies a non-trivial constraint on $\operatorname{Im} A_{3}\left(q^{2}, k^{2}, m\right)$ : this should be a smoothed delta-function with spreading parameter proportional to $k^{2}$ for $m^{2}=0$ (respectively, $m^{2}$ for $k^{2}=0$ ).

Let us summarize a number of facts which will also occur in the analysis of $\langle T T T\rangle$ :

- There could be algebraic degeneracies among the tensor structures which can be written for the three-point function.

- The anomaly can be expressed in terms of a finite, scheme-independent amplitude of the three-point function (i.e. $\left.A_{3}\left(q^{2}, 0\right) \propto C / q^{2}\right)$; this relation is analogous to $\langle T(z) T(0)\rangle=c / 2 z^{4}$ in two dimensions.

- Scheme-dependent amplitudes (i.e. $A_{1}$ ) are related to scheme-independent, finite amplitudes (i.e. $A_{3}$ ) by the Ward identities.

- The sum rule for the chiral anomaly (2.17) is similar to the sum rule (1.2) for the conformal anomaly in two dimensions, which is a version of the $c$-theorem: both expressions involve dimensionless densities which are smoothed delta-functions. This similarity will be fully explained in Section 4, where the sum rules for the fourdimensional trace anomalies will be derived by combining both arguments. 


\subsection{Definition of the stress tensor and Ward identities}

Here we consider a four-dimensional renormalizable field theory and suppose that it can be covariantly extended on classical curved-space backgrounds; at critical points, we also assume that the theory is covariant under Weyl transformations of the background metric $g_{\mu \nu}(x)$. The stress-tensor $n$-point function is defined by the following variation of the generating functional $W[g] \equiv \log Z[g]$ w.r.t. the metric:

$$
\left\langle T_{\mu_{1} \mu_{1}^{\prime}}\left(x_{1}\right) \cdots T_{\mu_{n} \mu_{n}^{\prime}}\left(x_{n}\right)\right\rangle \equiv \frac{1}{\sqrt{g\left(x_{1}\right)} \cdots \sqrt{g\left(x_{n}\right)}} \frac{\delta}{\delta g^{\mu_{1} \mu^{\prime}{ }_{1}\left(x_{1}\right)}} \cdots \frac{\delta}{\delta g^{\mu_{n} \mu^{\prime}{ }_{n}\left(x_{n}\right)}} W[g] .
$$

In this Equation, the $\sqrt{g}$ terms are pulled out of the variations, so that $n$-point functions explicitly respect the symmetry under exchanges of two stress tensors. Other definitions would yield $n$-point functions differing by lower $k$-point functions, $0 \leq k<n$; this is an intrinsic ambiguity of the stress-tensor which should be fixed together with the renormalization conditions (this point will be further discussed in Section 5).

The symmetries of coordinate reparametrizations (Diff) and, at critical points, of Weyl rescalings of the metric imply Ward identities which can be obtained by varying $W[g]$ and by consistently using the definition of the stress tensor (2.19) (see Appendix A for the conventions of curved-space calculus and Appendix B for a complete discussion of the Ward identities). We can write:

$$
\begin{aligned}
\text { Diff : } \delta_{\epsilon} W[g] & \equiv \int d x\left(D^{\mu} \epsilon^{\nu}(x)+D^{\nu} \epsilon^{\mu}(x)\right) \frac{\delta}{\delta g^{\mu \nu}(x)} W[g]=0 ; \\
\text { Weyl : } \delta_{\sigma} W[g] & \equiv-\int d x 2 \sigma(x) g^{\mu \nu}(x) \frac{\delta}{\delta g^{\mu \nu}(x)} W[g] \\
& =-\int d x 2 \sigma(x) \sqrt{g} \mathcal{A}(x ; g) .
\end{aligned}
$$

Note that the Weyl symmetry is anomalous with trace anomaly $\mathcal{A}(x ; g)$. The first variation w.r.t. $g_{\mu \nu}$ of the identities (2.20) yield the Equations:

Diff : $\quad D^{\mu}\left\langle T_{\mu \mu^{\prime}}\right\rangle=0$;

Weyl : $\quad g^{\mu \mu^{\prime}}\left\langle T_{\mu \mu^{\prime}}\right\rangle \equiv\langle\Theta\rangle=\mathcal{A}(x ; g)=\lambda\left(a E-3 c W+a^{\prime} D^{2} \mathcal{R}+r \mathcal{R}^{2}\right)$.

The Ward identities for multi-point functions are obtained by further variations w.r.t. $g^{\mu \mu^{\prime}}(x)$ of the Eqs. (2.21) (2.22).

The expression (2.22) of the trace does not contain explicit dynamical fields which would violate the Weyl symmetry at the classical level $\left(\Theta_{\text {classical }} \equiv 0\right)$ : the underlying hypothesis is that the scale-invariant theory in flat space can be extended to a Weylinvariant theory in curved space. Above two dimensions, this extension is not automatic: 
flat-space scale invariance is compatible with derivative fields in the trace, which, however, can often be removed by redefining (i.e. "improving") the stress tensor [28] (obstructions to improvement are discussed, e.g., in Ref. [5]).

\subsection{The form of $\langle T T\rangle$ and its scheme dependencies}

The general form of the stress-tensor two-point function is one of the ingredients which are needed for the construction of the three-point function: we recall here the analysis of Ref. [5], which can also be found in [13] 25]. From the variation of (2.21), we obtain the diffeomorphism Ward identity for the two-point function in flat space:

$$
2 \frac{\partial}{\partial y_{\mu}}\left\langle T_{\mu \nu}(y) T_{\alpha \beta}(x)\right\rangle+\frac{\partial}{\partial y^{(\alpha}}\left(\delta(y-x)\left\langle T_{\beta) \nu}(x)\right\rangle\right)+\left(\frac{\partial}{\partial y^{\nu}} \delta(y-x)\right)\left\langle T_{\alpha \beta}(x)\right\rangle=0,
$$

where the symmetrization of indices is $x^{(\mu} y^{\nu)}=x^{\mu} y^{\nu}+y^{\mu} x^{\nu}$. The standard normalization condition would fix the constant $\left\langle T_{\mu \nu}\right\rangle=0$ in flat space; at critical points, this is consistent with the absence of dimensionful parameters in the trace anomaly, such as a cosmological constant. On the other hand, we prefer to keep a non-vanishing one-point function for the later purpose of studying the scheme-dependence of $\langle T T T\rangle$ due to off-critical mass parameters; thus, $\left\langle T_{\mu \nu}\right\rangle \propto \eta_{\mu \nu} m^{4}$.

The first variation of (2.22) similarly yields the Ward identity for the Weyl symmetry at criticality:

$$
\left\langle\Theta(y) T_{\alpha \beta}(x)\right\rangle=\lambda a^{\prime}\left(\eta_{\alpha \beta} \partial^{2}-\partial_{\alpha} \partial_{\beta}\right) \partial^{2} \delta(x-y), \quad \text { (critical points). }
$$

It is convenient to contract the indices of the stress-tensor with generic polarization tensors $h_{\mu \nu}=h_{\nu \mu}$, and use the following notations:

$$
h_{\mu}^{\mu} \equiv(h), \quad\left(h_{1}\right)_{\alpha \beta}\left(h_{2}\right)^{\alpha \beta} \equiv\left(h_{1} . h_{2}\right), \quad p^{\alpha} h_{\alpha \beta} q^{\beta} \equiv(p . h . q) .
$$

The conservation and trace equations in momentum space will be obtained by respectively choosing the polarizations:

$$
h_{i} \rightarrow \frac{1}{2}\left(v \otimes k_{i}+k_{i} \otimes v\right), \quad h_{i} \rightarrow \eta, \quad i=1,2,
$$

where $v$ is an arbitrary four-vector, $k_{i}$ is the momentum of the Fourier transformed tensor $T\left(k_{i}\right)$ and $\eta$ the Euclidean (Minkowskian) metric (our conventions are listed in Appendix A).

In conclusion, the Diff and Weyl Ward identities for the two-point function in momentum space read:

Diff : $\left\langle(v \otimes p \cdot T(p))\left(h_{2} \cdot T(-p)\right)\right\rangle+2\left\langle\left(p \cdot h_{2} \cdot T \cdot v\right)\right\rangle+(v \cdot p)\left\langle\left(h_{2} \cdot T\right)\right\rangle=0$,

Weyl : $\left\langle\Theta(p)\left(h_{2} \cdot T_{2}(-p)\right)\right\rangle=\lambda a^{\prime} p^{2}\left(p^{2}\left(h_{2}\right)-\left(p \cdot h_{2} \cdot p\right)\right), \quad$ (critical points). 
The general form of the two-point function in momentum space is obtained by the procedure of Section 2.1: the general expansion in tensor structures times scalar amplitudes is constrained by the Ward identities. The one-point function can be written:

$$
\langle(h . T)\rangle=-2(h) f_{\Lambda},
$$

where $f_{\Lambda}$ is a dimensionful constant related to the mass. The two-point function solution of the Diff Ward identity (2.27) is found to be [5]:

$$
\begin{aligned}
\left\langle\left(h_{1} \cdot T(p)\right)\right. & \left.\left(h_{2} \cdot T(-p)\right)\right\rangle \\
= & \left(f_{0}\left(p^{2}\right)-f_{2}\left(p^{2}\right)\right)\left(p^{2}\left(h_{1}\right)-\left(p \cdot h_{1} \cdot p\right)\right)\left(p^{2}\left(h_{2}\right)-\left(p \cdot h_{2} \cdot p\right)\right) \\
+ & (d-1) f_{2}\left(p^{2}\right)\left[\left(p \cdot h_{1} \cdot p\right)\left(p \cdot h_{2} \cdot p\right)-2 p^{2}\left(p \cdot h_{1} \cdot h_{2} \cdot p\right)+p^{4}\left(h_{1} \cdot h_{2}\right)\right] \\
+ & f_{\Lambda}\left[\left(h_{1}\right)\left(h_{2}\right)+2\left(h_{1} \cdot h_{2}\right)\right] .
\end{aligned}
$$

The two independent scalar amplitudes $f_{0}\left(p^{2}\right)$ and $f_{2}\left(p^{2}\right)$ are dimensionless functions of the squared momentum and the mass; they parameterize the propagation of intermediate states of spin zero and two, respectively, and are multiplied by transverse tensors. Moreover, the spin-two tensor is traceless (in two dimensions, it vanishes identically providing another example of tensor degeneracy). For later purposes, it is convenient to write the solution for general space-time dimension $d$, and set $d=4$ when comparing with the trace anomaly.

At the critical points $(m=0)$, the two dimensionless amplitudes can depend logarithmically on the momentum or be constant: the spin-zero amplitude is completely determined by the Weyl Ward identity (2.28):

$$
f_{0}^{c}\left(p^{2}\right)=\frac{\lambda a^{\prime}}{d-1}, \quad \text { (critical points). }
$$

The absence of a logarithmic term is due to our starting hypothesis of Weyl invariance at criticality, which forces the trace for the stress tensor to vanish as an operator; its correlators can only contain scheme-dependent contact terms. Thus, the spin-zero part of $\langle T T\rangle$ is a polynomial of momenta of dimension four which can be modified by adding counterterms to the effective action. This verifies the well-known scheme dependence $a^{\prime} \rightarrow a^{\prime}+$ const in the trace anomaly (1.1) 13.

The amplitude $f_{2}^{c}\left(p^{2}\right)$ has instead a logarithmic singularity at the bare level; its renormalized expression is:

$$
f_{2}^{c}\left(p^{2}\right)=-\lambda c \log \left(\frac{p^{2}}{\mu^{2}}\right), \quad \text { (critical points). }
$$

In the above Equation, $\mu$ is the renormalization scale and $c$ is the coefficient of the Weyl term in the trace anomaly (2.22): the latter fact can be proved [13] [5 by using the Ward 
identity for scale invariance in flat space (a conformal isometry fully discussed in Section 3.3). Equation (2.32) shows that $f_{2}^{c}\left(p^{2}\right)$ is defined up to a scheme-dependent constant, while the coefficient $c$ is scheme-independent: its value normalizes the two-point function at criticality (for non-coincident coordinates, $x_{1} \neq x_{2}$ ) and is positive for unitary theories.

In conclusion, the two $\langle T T\rangle$ amplitudes depend on the renormalization scheme by constant shifts, which change the correlator by polynomials of the momenta. The schemedependence of any correlator can be characterized as follows: renormalization theory says that counterterms in the action are polynomial of momenta with maximal degree $d$, thus scheme-dependent terms of a $\Delta$-dimensional $n$-point function $\left\langle\mathcal{O}_{1}\left(k_{1}\right) \cdots \mathcal{O}_{n}\left(k_{n}\right)\right\rangle$ are polynomials of maximal dimension $\Delta(\Delta>0$ is assumed here). These polynomials can be added to the $n$-point function, which is being expanded in tensor structures times scalar amplitudes, as done for $\langle A V V\rangle$ and $\langle T T\rangle$; if a polynomial matches one of the tensors in the expansion, the corresponding scalar amplitude is redefined.

This scheme dependence can be characterized by a number associated to the corresponding tensor structure, which will be called the tensorial dimension $\delta_{T}$. This is defined as the highest number of free-index momenta of the monomials contained in the tensor (as usual, free indices are contracted with polarizations $h_{i}$ or $v$ ). For instance, $\delta_{T}\left[\left(k_{1} \cdot h_{2} \cdot k_{2}\right)\left(k_{2} \cdot h_{1} \cdot k_{2}\right)+k^{2} q^{2}\left(h_{1} \cdot h_{2}\right)\right]=4$ and $\delta_{T}\left[k^{2} q^{2}\left(h_{1} \cdot h_{2}\right)\right]=0$. Therefore, the $f_{0}, f_{2}$ amplitudes in (2.30) are both associated to $\delta_{T}=4$ tensors, while $f_{\Lambda}$ multiplies a $\delta_{T}=0$ expression.

A sufficient condition for an amplitude to be scheme independent is that its tensor has dimension $\delta_{T}>\Delta$ : clearly, tensors with $\delta_{T}>\Delta$ cannot mix with counterterms, while $\delta_{T} \leq \Delta$ tensors could mix with them - a detailed analysis is then necessary. For the $n$-point functions of the stress tensor $(\Delta=d)$, the scheme-independence condition is $\delta_{T}>4$ in four dimensions; all amplitudes in $\langle T T\rangle$ have associated tensors with $\delta_{T} \leq 4$ and are actually scheme-dependent. On the contrary, $\langle T T T\rangle$ can have $\delta_{T}=6$ tensors and corresponding finite, scheme-independent amplitudes; finding the finite amplitudes related to the anomaly coefficients $a$ and $c$ is the main motivation for studying the three-point function.

\section{The stress-tensor three-point function}




\subsection{Derivation of the general form}

In this Section, we derive the general form of the stress-tensor three-point function at the symmetric point $k^{2} \equiv k_{1}^{2}=k_{2}^{2}$ :

$$
\left\langle\left(h_{3} \cdot T\left(k_{3}\right)\right)\left(h_{2} \cdot T\left(k_{2}\right)\right)\left(h_{1} \cdot T\left(k_{1}\right)\right)\right\rangle, \quad q^{\mu} \equiv-k_{3}^{\mu}=k_{1}^{\mu}+k_{2}^{\mu}, \quad k_{1}^{2}=k_{2}^{2} \equiv k^{2} .
$$

Although the point $q^{2}=k^{2}$ is the most symmetric, it will be not consider here because it lays in the unphysical region of complex momenta, while the choice $q^{2} \neq k^{2}$ allows for real (Minkowskian) momenta both at and off criticality.

The diffeomorphism Ward identity constraints the longitudinal component of the stress tensors: for $T\left(k_{3}\right)$, it reads (see Appendix $\mathrm{B}$ for the derivation):

$$
\begin{aligned}
0 & =2\left\langle\left(k_{3} \cdot T\left(k_{3}\right) \cdot v\right)\left(T\left(k_{2}\right) \cdot h_{2}\right)\left(T\left(k_{1}\right) \cdot h_{1}\right)\right\rangle \\
& +2\left\langle\left(k_{3} \cdot h_{2} \cdot T\left(-k_{1}\right) \cdot v\right)\left(T\left(k_{1}\right) \cdot h_{1}\right)\right\rangle-\left(k_{2} \cdot v\right)\left\langle\left(T\left(-k_{1}\right) \cdot h_{2}\right)\left(T\left(k_{1}\right) \cdot h_{1}\right)\right\rangle \\
& +2\left\langle\left(k_{3} \cdot h_{1} \cdot T\left(-k_{2}\right) \cdot v\right)\left(T\left(k_{2}\right) \cdot h_{2}\right)\right\rangle-\left(k_{1} \cdot v\right)\left\langle\left(T\left(-k_{2}\right) \cdot h_{1}\right)\left(T\left(k_{2}\right) \cdot h_{2}\right)\right\rangle .
\end{aligned}
$$

Note that this inhomogeneous equation relates the three-point function to the two-point correlator already found in Section 2.3.

The general form of $\langle T T T\rangle$ off criticality can be expanded over the basis of the Lorentz tensors which are built out of $k_{1}^{\alpha}, k_{2}^{\beta}, \eta^{\mu \nu}$ : there are 137 elementary polynomials $\mathcal{P}_{i}=$ $\mathcal{P}_{i}\left(k_{1}^{\alpha}, k_{2}^{\beta}, \eta^{\mu \nu}\right)$ of maximal tensorial dimension $\delta_{T}=6$, which are classified in Appendix C. After imposing the Bose symmetry $\left(h_{1}, k_{1}\right) \leftrightarrow\left(h_{2}, k_{2}\right)$ at the symmetric point $k_{1}^{2}=k_{2}^{2}$, the number of independent polynomials reduces to 77 (see Table 1). This general expansion is plugged into the Ward identity (3.2) and the corresponding one for $\left(k_{1} \leftrightarrow k_{3}\right)$, which yield linear systems for the scalar amplitudes (the algebra is done with the help of Mathematica [26] routines). In order to avoid the problem of possible degeneracies of the tensor basis due to relations proper to four-dimensions, we solve the system in arbitrary large dimensions, and postpone the discussion of the limit to four dimension. The solution of the Ward identity (3.2) and its $(1 \leftrightarrow 3)$ analogue contains 17 transverse tensors, solutions of the homogeneous equations, plus 5 inhomogeneous (i.e. non transverse) tensors multiplying

the two- and one-point amplitudes $\left\{f_{0}\left(q^{2}\right), f_{0}\left(k^{2}\right), f_{2}\left(q^{2}\right), f_{2}\left(k^{2}\right), f_{\Lambda}\right\}$ already found in Section 2.3:

$$
\begin{aligned}
\left\langle\left(h_{1} . T\right)\left(h_{2} . T\right)\left(h_{3} . T\right)\right\rangle & =\sum_{i=1}^{17} A_{i}\left(q^{2}, k^{2}\right) \mathcal{T}_{i} \\
& +f_{0}\left(q^{2}\right) \mathcal{T}_{0 q}+f_{0}\left(k^{2}\right) \mathcal{T}_{0 k}+f_{2}\left(q^{2}\right) \mathcal{T}_{2 q}+f_{2}\left(k^{2}\right) \mathcal{T}_{2 k} \\
& +f_{\Lambda} \mathcal{T}_{\Lambda} .
\end{aligned}
$$

Note that the tensor structures are contracted with the polarizations $\left(h_{1}, h_{2}, h_{3}\right)$, i.e. $\mathcal{T}_{\omega}=\mathcal{T}_{\omega}\left(k_{1}, h_{1}, k_{2}, h_{2}, h_{3}\right)$, for $\omega=1, \ldots, 17,0 q, 0 k, 2 q, 2 k, \Lambda$. 


\begin{tabular}{|c||c|c|c|c|}
\hline $\mathbf{N}$ & $\mathbf{N + 1}$ & $\mathbf{N + 2}$ & $\mathbf{N}+\mathbf{3}$ & $\mathbf{N + 4}$ \\
\hline \hline 0 & $(1|1| 1)(1|2| 1)(1|3| 1)$ & $(1|3| 1)(2|1| 2)(2|2| 2)$ & $(1|1| 1)(1|2| 1)(1|3| 2)$ & $(1|1| 1)(1|3| 1)(2|2| 2)$ \\
\hline 4 & $(1|1| 1)(1|3| 2)(2|2| 2)$ & $(1|1| 1)(1|3| 1)(1|2| 2)$ & $(1|3| 1)(1|1| 2)(2|2| 2)$ & $(1|1| 1)(1|2| 2)(1|3| 2)$ \\
\hline 8 & $(1|2| 1)(1|3| 1)(2|1| 2)$ & $(1|2| 1)(1|3| 2)(2|1| 2)$ & $(1|3| 1)(1|2| 2)(2|1| 2)$ & $(1|2| 1)(1|3| 1)(1|1| 2)$ \\
\hline 12 & $(1|2| 1)(1|1| 2)(1|3| 2)$ & $(1|3| 1)(1|1| 2)(1|2| 2)$ & $(1|1| 2)(1|2| 2)(1|3| 2)$ & $(1|1| 1)(1|32| 1)$ \\
\hline 16 & $(1|31| 1)(2|2| 2)$ & $(1|1| 1)(1|32| 2)$ & $(1|1| 1)(2|32| 1)$ & $(1|32| 1)(2|1| 2)$ \\
\hline 20 & $(1|2| 1)(1|31| 1)$ & $(1|2| 1)(2|31| 1)$ & $(1|2| 1)(1|31| 2)$ & $(1|32| 1)(1|1| 2)$ \\
\hline 24 & $(1|31| 1)(1|2| 2)$ & $(1|1| 2)(1|32| 2)$ & $(1|2| 2)(1|31| 2)$ & $(1|3| 1)(1|21| 1)$ \\
\hline 28 & $(1|21| 1)(2|3| 2)$ & $(1|3| 1)(1|21| 2)$ & $(1|3| 1)(2|21| 1)$ & $(1|21| 1)(1|3| 2)$ \\
\hline 32 & $(1|3| 2)(1|21| 2)$ & $(1|3| 2)(2|21| 1)$ & $(3)(1|1| 1)(1|2| 1)$ & $(3)(1|1| 1)(2|2| 2)$ \\
\hline 36 & $(3)(1|1| 1)(1|2| 2)$ & $(2)(1|1| 1)(1|3| 1)$ & $(1)(1|3| 1)(2|2| 2)$ & $(2)(1|1| 1)(1|3| 2)$ \\
\hline 40 & $(3)(1|2| 1)(2|1| 2)$ & $(3)(1|2| 1)(1|1| 2)$ & $(2)(1|3| 1)(2|1| 2)$ & $(1)(1|2| 1)(1|3| 1)$ \\
\hline 44 & $(1)(1|2| 1)(1|3| 2)$ & $(3)(1|1| 2)(1|2| 2)$ & $(2)(1|3| 1)(1|1| 2)$ & $(1)(1|3| 1)(1|2| 2)$ \\
\hline 48 & $(2)(1|1| 2)(1|3| 2)$ & $(1|312| 1)$ & $(1|321| 1)$ & $(1|231| 1)$ \\
\hline 52 & $(1|312| 2)$ & $(1|321| 2)$ & $(1|231| 2)$ & $(2|231| 1)$ \\
\hline 56 & $(32)(1|1| 1)$ & $(31)(1|2| 1)$ & $(32)(1|1| 2)$ & $(1)(1|32| 1)$ \\
\hline 60 & $(2)(1|31| 1)$ & $(1)(1|32| 2)$ & $(2)(1|31| 2)$ & $(21)(1|3| 1)$ \\
\hline 64 & $(21)(1|3| 2)$ & $(3)(1|21| 1)$ & $(3)(1|21| 2)$ & $(3)(2|21| 1)$ \\
\hline 68 & $(2)(3)(1|1| 1)$ & $(1)(3)(1|2| 1)$ & $(2)(3)(1|1| 2)$ & $(1)(2)(1|3| 1)$ \\
\hline 72 & $(1)(2)(1|3| 2)$ & $(321)$ & $(2)(31)$ & $(3)(21)$ \\
\hline 76 & $(1)(2)(3)$ & & & \\
\hline
\end{tabular}

Table 1: The basis of $(1 \leftrightarrow 2)$ symmetric six-index polynomials of $k_{1}^{\mu}, k_{2}^{\nu}$ and $\eta_{\alpha \beta}$ : $\mathcal{P}_{i}=\mathcal{P}_{i}\left(k_{1}, h_{1}, k_{2}, h_{2}, h_{3}\right), i=1, \ldots, 77$. We use the short-hand notations: $(i|a b c| j) \equiv$ $k_{i} \cdot h_{a} \cdot h_{b} \cdot h_{c} \cdot k_{j}, \ldots, \quad(i \mid j) \equiv k_{i} \cdot k_{j}(a b c) \equiv \operatorname{tr}\left(h_{a} \cdot h_{b} \cdot h_{c}\right), \ldots,(a) \equiv \operatorname{tr}\left(h_{a}\right)$ and we omit the $(1 \leftrightarrow 2)$ exchanged term that must be added to all non $(1 \leftrightarrow 2)$ symmetric polynomials of the table. The tensorial dimension of the polynomial $\mathcal{P}_{i}$ is: $\delta_{T}=6$ for $i=1, \ldots, 15$, $\delta_{T}=4$ for $i=16, \ldots, 49 ; \delta_{T}=2$ for $i=50, \ldots, 73$ and $\delta_{T}=0$ for $i=74, \ldots, 77$. 
At criticality we must impose the Ward identities for the Weyl symmetry, which compare $\langle T T T\rangle$ traced over one of the stress tensors with the second variation of the anomaly (2.22). There are two independent equations by tracing $T\left(k_{3}\right)$ or $T\left(k_{1}\right)$; in the first case, we find (see Appendix B):

$$
\begin{aligned}
& \left\langle\Theta\left(k_{3}\right)\left(T\left(k_{1}\right) \cdot h_{1}\right)\left(T\left(k_{2}\right) \cdot h_{2}\right)\right\rangle \\
& +\left[\left\langle\left(T\left(-k_{2}\right) \cdot h_{1}\right)\left(T\left(k_{2}\right) \cdot h_{2}\right)\right\rangle+\left\langle\left(T\left(k_{1}\right) \cdot h_{1}\right)\left(T\left(-k_{1}\right) \cdot h_{2}\right)\right\rangle\right] \\
& =\mathcal{A}\left(k_{1}, h_{1} ; k_{2}, h_{2}\right)-\frac{1}{2}\left[\left(h_{1}\right) \mathcal{A}\left(k_{2}, h_{2}\right)+\left(h_{2}\right) \mathcal{A}\left(k_{1}, h_{1}\right)\right] .
\end{aligned}
$$

In this Equation, $\mathcal{A}\left(k_{1}, h_{1}\right)$ and $\mathcal{A}\left(k_{1}, h_{1} ; k_{2}, h_{2}\right)$ are, respectively, the first and second variation of the anomaly (2.22) w.r.t. to the metric, $\left(h_{i} . \delta / \delta g\left(k_{i}\right)\right)$, evaluated in flat space. Their explicit expressions are:

$$
\mathcal{A}\left(k_{1}, h_{1}\right) / \lambda=a^{\prime} D^{2} \mathcal{R}\left(k_{1}, h_{1}\right)=a^{\prime} k_{1}^{2}\left(k_{1}^{2}\left(h_{1}\right)-\left(k_{1} \cdot h_{1} \cdot k_{1}\right)\right) ;
$$

and

$$
\begin{aligned}
\mathcal{A}\left(k_{1}, h_{1} ; k_{2}, h_{2}\right) / \lambda & =(-3 c+a)\left(R_{\mu \nu \alpha \beta}\right)^{2}\left(k_{1}, h_{1} ; k_{2}, h_{2}\right) \\
& +(6 c-4 a)\left(\mathcal{R}_{\alpha \beta}\right)^{2}\left(k_{1}, h_{1} ; k_{2}, h_{2}\right) \\
& +(-c+a+r) \mathcal{R}^{2}\left(k_{1}, h_{1}, k_{2}, h_{2}\right) \\
& +a^{\prime} D^{2} \mathcal{R}\left(k_{1}, h_{1} ; h_{2}, k_{2}\right)
\end{aligned}
$$

with

$$
\begin{aligned}
\left(R_{\mu \nu \alpha \beta}\right)^{2}\left(k_{1}, h_{1} ; k_{2}, h_{2}\right)= & 2\left[\left(k_{1} \cdot k_{2}\right)^{2}\left(h_{1} \cdot h_{2}\right)+\left(k_{1} \cdot h_{2} \cdot k_{1}\right)\left(k_{2} \cdot h_{1} \cdot k_{2}\right)\right. \\
\left(\mathcal{R}_{\alpha \beta}\right)^{2}\left(k_{1}, h_{1} ; k_{2}, h_{2}\right)= & \left.-2\left(k_{1} \cdot k_{2}\right)\left(k_{1} \cdot h_{2} \cdot h_{1} \cdot k_{2}\right)\right] \\
& \frac{1}{2}\left(k_{1} \cdot k_{2}\right)^{2}\left(h_{1}\right)\left(h_{2}\right)+\frac{1}{2} k_{1}^{2} k_{2}^{2}\left(h_{1} \cdot h_{2}\right) \\
& +\left(k_{1} \cdot k_{2}\right)\left(k_{1} \cdot h_{1} \cdot h_{2} \cdot k_{2}\right)+\left(k_{2} \cdot h_{2} \cdot k_{1}\right)\left(k_{1} \cdot h_{1} \cdot k_{2}\right) \\
& +\left[\frac{1}{2} k_{2}^{2}\left(h_{1}\right)\left(k_{1} \cdot h_{2} \cdot k_{1}\right)-\left(k_{1} \cdot k_{2}\right)\left(h_{1}\right)\left(k_{2} \cdot h_{2} \cdot k_{1}\right)\right. \\
& \left.-k_{1}^{2}\left(k_{2} \cdot h_{2} \cdot h_{1} \cdot k_{2}\right)+\{1 \leftrightarrow 2\}\right], \\
\mathcal{R}^{2}\left(k_{1}, h_{1} ; k_{2}, h_{2}\right)= & 2\left[\left(k_{1}^{2}\left(h_{1}\right)-\left(k_{1} \cdot h_{1} \cdot k_{1}\right)\right)(\{1 \leftrightarrow 2\})\right], \\
D^{2} \mathcal{R}\left(k_{1}, h_{1} ; k_{2}, h_{2}\right)= & {\left[\left(k_{1}^{2}\left(h_{1}\right)-\left(k_{1} \cdot h_{1} \cdot k_{1}\right)\right)\right.} \\
& \left.\times\left(\left(k_{1} \cdot h_{2} \cdot k_{1}\right)+\left(k_{2} \cdot h_{2} \cdot k_{1}\right)-\frac{1}{2}\left(k_{1} \cdot k_{2}\right)\left(h_{2}\right)\right)+(\{1 \leftrightarrow 2\})\right] \\
& +\left(k_{1}+k_{2}\right)^{2}\left[-\left(h_{1} \cdot h_{2}\right)\left(k_{1}^{2}+k_{2}^{2}+\frac{5}{2}\left(k_{1} \cdot k_{2}\right)\right)-\frac{1}{2}\left(k_{1} \cdot k_{2}\right)\left(h_{1}\right)\left(h_{2}\right)\right. \\
& +\left(k_{2} \cdot h_{1} \cdot h_{2} \cdot k_{1}\right)+\left(h_{1}\right)\left(\left(k_{1} \cdot h_{2} \cdot k_{1}\right)+\left(k_{1} \cdot h_{2} \cdot k_{2}\right)\right) \\
& \left.+\left(h_{2}\right)\left(\left(k_{2} \cdot h_{1} \cdot k_{2}\right)+\left(k_{2} \cdot h_{1} \cdot k_{1}\right)\right)\right] \cdot \\
&
\end{aligned}
$$

The consistency among the two Weyl Ward identities implies the vanishing of the coefficient $r$ in the trace anomaly (1.1) at criticality; this is the Wess-Zumino condition, which requires the commutativity of Weyl variations [12]. Furthermore, the consistencies between Weyl and Diff identities imply $f_{0}^{c}\left(q^{2}\right)=f_{0}^{c}\left(k^{2}\right)=\lambda a^{\prime} / 3$ in agreement with the earlier result (2.31). 
Next, the two Weyl Ward identities are imposed on the form of $\langle T T T\rangle$ satisfying the diffeomorphism identities, Eq.(3.3); a solution is found in four dimensions only, and the number of independent tensor structures reduces from $22=(17$ transverse +5 non-transverse $)$ to $13=(8$ transverse-traceless +2 transverse-traceful +3 non-transverse-traceful $)$. The final result for $\langle T T T\rangle$ off-criticality is thus given by (3.3) up to a convenient change of basis, which makes the critical limit simpler and also fulfills other requirements to be explained later:

$$
\begin{aligned}
\left\langle\left(h_{1} . T\right)\left(h_{2} . T\right)\left(h_{3} . T\right)\right\rangle & =\sum_{i=1}^{8} A_{i}\left(q^{2}, k^{2}\right) \mathcal{T}_{i}+A_{E}\left(q^{2}, k^{2}\right) \mathcal{T}_{E}+A_{W}\left(q^{2}, k^{2}\right) \mathcal{T}_{W} \\
& +\sum_{i=11}^{17} A_{i}\left(q^{2}, k^{2}\right) \mathcal{T}_{i} \\
& +\sum_{ \pm}\left[f_{0 \pm}\left(q^{2}, k^{2}\right) \mathcal{T}_{0 \pm}+f_{2 \pm}\left(q^{2}, k^{2}\right) \mathcal{T}_{2 \pm}\right]+f_{\Lambda} \mathcal{T}_{\Lambda}
\end{aligned}
$$

In this Equation, the 22 tensors $\mathcal{T}_{\omega}$ only depend on the external momenta and do not contain any parameter of the theory, thus they are invariant of the renormalization-group flow and provide a convenient basis both at and off criticality. The scalar amplitudes $A_{i}$ carry all the dependence on the flow: at criticality, some of them are constrained by the Weyl identities, in particular $9=22-13$ of them vanish $(7$ transverse-traceful +2 nontransverse-traceful). This is made manifest in the basis in Eq. (3.8), which is an extension of the basis at criticality.

More specifically, the 22 tensors have the following properties (see also Table 2):

- The tensors $\mathcal{T}_{i}, i=1, \ldots, 8$ are transverse and traceless w.r.t. all the three stresstensors and thus the corresponding amplitudes are unconstrained at criticality.

- The tensors $\mathcal{T}_{E} \equiv \mathcal{T}_{9}$ and $\mathcal{T}_{W} \equiv \mathcal{T}_{10}$ are transverse and traceful; their traces match the second variation (3.7) of the Euler and Weyl-square terms in the anomaly (1.1), respectively; the corresponding amplitudes have the critical limits:

$$
\begin{aligned}
& A_{E}\left(q^{2}, k^{2}\right) \rightarrow A_{E}^{c}\left(q^{2}, k^{2}\right)=\lambda \frac{a}{q^{2}} \\
& A_{W}\left(q^{2}, k^{2}\right) \rightarrow A_{W}^{c}\left(q^{2}, k^{2}\right)=\lambda \frac{c}{q^{2}}, \quad \text { (critical points). }
\end{aligned}
$$

- The seven tensors $\mathcal{T}_{i}, i=11, \ldots, 17$ are transverse and traceful, but their traces do not match any of the terms in the anomaly (1.1). Therefore, their amplitudes should vanish at criticality:

$$
A_{i}\left(q^{2}, k^{2}\right) \rightarrow 0, \quad i=11, \ldots, 17, \quad \text { (critical points). }
$$




\begin{tabular}{|c||c|c|c|c|c|c|}
\hline & $\perp k_{1,2}$ & $\perp k_{3}$ & $\operatorname{tr}_{1,2}=0$ & $\operatorname{tr}_{3}=0$ & $\delta_{T}$ & $\Delta$ \\
\hline \hline $\mathcal{T}_{1}$ & $\mathrm{y}$ & $\mathrm{y}$ & $\mathrm{y}$ & $\mathrm{y}$ & 6 & $>6$ \\
\hline $\mathcal{T}_{2}$ & $\mathrm{y}$ & $\mathrm{y}$ & $\mathrm{y}$ & $\mathrm{y}$ & 6 & $>6$ \\
\hline $\mathcal{T}_{3}$ & $\mathrm{y}$ & $\mathrm{y}$ & $\mathrm{y}$ & $\mathrm{y}$ & 6 & $>6$ \\
\hline $\mathcal{T}_{4}$ & $\mathrm{y}$ & $\mathrm{y}$ & $\mathrm{y}$ & $\mathrm{y}$ & 6 & $>6$ \\
\hline $\mathcal{T}_{5}$ & $\mathrm{y}$ & $\mathrm{y}$ & $\mathrm{y}$ & $\mathrm{y}$ & 6 & $>6$ \\
\hline $\mathcal{T}_{6}$ & $\mathrm{y}$ & $\mathrm{y}$ & $\mathrm{y}$ & $\mathrm{y}$ & 6 & $>6$ \\
\hline $\mathcal{T}_{7}$ & $\mathrm{y}$ & $\mathrm{y}$ & $\mathrm{y}$ & $\mathrm{y}$ & 6 & $>6$ \\
\hline $\mathcal{T}_{8}$ & $\mathrm{y}$ & $\mathrm{y}$ & $\mathrm{y}$ & $\mathrm{y}$ & 4 & 4 \\
\hline \hline $\mathcal{T}_{E}$ & $\mathrm{y}$ & $\mathrm{y}$ & $\mathrm{n}$ & $\mathrm{n}$ & 6 & 6 \\
\hline $\mathcal{T}_{W}$ & $\mathrm{y}$ & $\mathrm{y}$ & $\mathrm{n}$ & $\mathrm{n}$ & 6 & 8 \\
\hline \hline $\mathcal{T}_{11}$ & $\mathrm{y}$ & $\mathrm{y}$ & $\mathrm{n}$ & $\mathrm{n}$ & 4 & 6 \\
\hline $\mathcal{T}_{12}$ & $\mathrm{y}$ & $\mathrm{y}$ & $\mathrm{n}$ & $\mathrm{n}$ & 4 & 6 \\
\hline $\mathcal{T}_{13}$ & $\mathrm{y}$ & $\mathrm{y}$ & $\mathrm{n}$ & $\mathrm{n}$ & 6 & 6 \\
\hline $\mathcal{T}_{14}$ & $\mathrm{y}$ & $\mathrm{y}$ & $\mathrm{n}$ & $\mathrm{n}$ & 6 & 6 \\
\hline $\mathcal{T}_{15}$ & $\mathrm{y}$ & $\mathrm{y}$ & $\mathrm{n}$ & $\mathrm{n}$ & 6 & 6 \\
\hline $\mathcal{T}_{16}$ & $\mathrm{y}$ & $\mathrm{y}$ & $\mathrm{n}$ & $\mathrm{n}$ & 6 & 6 \\
\hline $\mathcal{T}_{17}$ & $\mathrm{y}$ & $\mathrm{y}$ & $\mathrm{n}$ & $\mathrm{n}$ & 6 & $>6$ \\
\hline \hline $\mathcal{T}_{0+}$ & $\mathrm{n}$ & $\mathrm{n}$ & $\mathrm{n}$ & $\mathrm{n}$ & 4 & 4 \\
\hline $\mathcal{T}_{0-}$ & $\mathrm{n}$ & $\mathrm{n}$ & $\mathrm{n}$ & $\mathrm{n}$ & 6 & $>6$ \\
\hline $\mathcal{T}_{2+}$ & $\mathrm{n}$ & $\mathrm{n}$ & $\mathrm{n}$ & $\mathrm{n}$ & 4 & 4 \\
\hline $\mathcal{T}_{2-}$ & $\mathrm{n}$ & $\mathrm{n}$ & $\mathrm{n}$ & $\mathrm{n}$ & 6 & 6 \\
\hline $\mathcal{T}_{\Lambda}$ & $\mathrm{n}$ & $\mathrm{n}$ & $\mathrm{n}$ & $\mathrm{n}$ & 0 & 4 \\
\hline \hline
\end{tabular}

Table 2: Properties of the tensors in Eq.(3.8); $(\perp)$ : transversality w.r.t. $k_{1}\left(k_{2}\right)$ and $k_{3}$ (yes/no); $(\operatorname{tr}=0)$ : tracelessness w.r.t. $T_{1}\left(T_{2}\right)$ and $T_{3} ; \delta_{T}$ : tensorial dimension; $\Delta$ : scale dimension of the tensor in polynomial form. 
These amplitudes are nevertheless different from zero off criticality; another parameterization of $\langle\Theta\rangle$ off criticality has been given in Ref.[甘], but cannot be immediately compared with our result.

- The five tensors $\mathcal{T}_{i \pm}, i=0,2$, and $\mathcal{T}_{\Lambda}$ are neither transverse nor traceless; the first four are the even and odd combinations of the tensors multiplying the two-point amplitudes $f_{i}\left(q^{2}\right)$ and $f_{i}\left(k^{2}\right)$ (see Eq.(3.3)):

$$
\begin{aligned}
\mathcal{T}_{i \pm} & \equiv\left(\mathcal{T}_{i q} \pm \mathcal{T}_{i k}\right), \\
f_{i \pm} & \equiv \frac{1}{2}\left(f_{i}\left(q^{2}\right) \pm f_{i}\left(k^{2}\right)\right), \quad i=0,2 .
\end{aligned}
$$

The fifth tensor accounts for the one-point amplitude $f_{\Lambda}(2.29)$. The critical limits of these amplitudes are:

$$
\begin{aligned}
f_{0+}\left(q^{2}, k^{2}\right) & \rightarrow \lambda \frac{a^{\prime}}{3} \\
f_{0-}\left(q^{2}, k^{2}\right) & \rightarrow 0 \\
f_{2 \pm}\left(q^{2}, k^{2}\right) & \rightarrow-\lambda \frac{c}{2}\left(\log \left(\frac{q^{2}}{\mu^{2}}\right) \pm \log \left(\frac{k^{2}}{\mu^{2}}\right)\right) \\
f_{\Lambda} & \rightarrow 0,
\end{aligned}
$$

Actually, the solution of the Weyl identities (3.4) yields the results (3.13, 3.14), which agree with the earlier result (2.31); on the other hand, the spin-two amplitudes $f_{2 \pm}$ are not constrained by the Weyl identities and their critical values are taken from (2.32).

\subsection{Further properties and renormalization}

\section{Null tensor.}

The basis of elementary tensors $\left\{\mathcal{P}_{i}\right\}$ (Table 1) is degenerate in four dimensions by the same mechanism found for $\langle A V V\rangle$ (Eq.(2.4)) and the two-dimensional $\langle T T\rangle$ : there exists one "null tensor" which do not manifestly vanish in the tensor notation used here, but actually has all components equal to zero in four dimensions. Such six-index Bose-symmetric null vector was found by inspection and, moreover, no null vectors were found with five and four indices within the tensors bases used for the Diff and Weyl Ward identities, respectively. Therefore, the corresponding linear systems for the coefficients have been correctly extracted from faithful bases. The null tensor clearly satisfies all Ward identities and can be eliminated a-posteriori from the final result (3.8): it is the traceless-transverse tensor $\mathcal{T}_{8}$ (see Table 2), which will be discard hereafter (its expression is given in Appendix D). 


\section{Choice of basis for the tensors.}

Each of the tensors $\mathcal{T}_{\omega}$ can be considered as a vector $(u)$ in the 77 -dimensional $\mathcal{P}_{i}$-space (Table 1) and the Ward identities are inhomogeneous linear equations of the form $A$. $u=w$, whose solutions possess characteristic ambiguities: (I) the homogeneous solutions $u_{0}, A \cdot u_{0}=0$, are determined up to linear combinations and must be normalized; (II) the inhomogeneous solutions are defined up to the addition of homogeneous solutions: $u=A^{-1} \cdot w \rightarrow A^{-1} \cdot w+u_{0}$. Using this freedom, we can choose a basis of tensors $\mathcal{T}_{\omega}$ which is more suitable for the physical interpretation. Generically, the tensors have the form:

$$
\mathcal{T}_{\omega}=\sum_{i=1}^{77} Q_{i}^{\omega}\left(q^{2}, k^{2}\right) \mathcal{P}_{i}\left(k_{1}, h_{1}, k_{2}, h_{2}, h_{3}\right),
$$

where the $Q_{i}^{\omega}$ are rational functions of the Lorentz invariants with various scale dimensions (the $\mathcal{P}_{i}$ have dimension equal to their tensorial dimension, $\Delta=\delta_{T}=0,2,4,6$, listed in Table (1). Thus, the two ambiguities (I) and (II) can be used to find convenient forms for the $Q_{i}^{\omega}$.

Let us first consider the choice in which the $Q_{i}^{\omega}$ are polynomial: this is clearly possible for the homogeneous solutions of the Diff Ward identity $\left(\mathcal{T}_{i}, i=1, \ldots, 17\right)$, by factoring out the common denominators into the corresponding amplitudes. As a matter of fact, four of the inhomogeneous solutions (last five entries in Table 2) can also be put in polynomial form using type-(II) redefinitions and without changing their amplitudes, which are inputs of the Ward identities. The scale dimensions of the $\mathcal{T}_{\omega}$ 's in polynomial form are listed in the last column of Table 2; we find the following cases:

- The tensors with $\Delta=4$ and $\delta_{T} \leq 4$ are naturally polynomial because they have the same dimension as $\langle T T T\rangle$; the corresponding amplitudes are dimensionless functions (or constants), as in the case of the two-point function (Section 2.3).

- The polynomial form also makes sense for the tensors with $\Delta=6$ and $\delta_{T}=4,6$; their amplitudes have the natural dimension -2 and may display a simple pole in the massless theory.

- The polynomial form is not convenient for tensors with $\delta_{T}=6$ and $\Delta>6$; the corresponding amplitudes would have large negative dimensions $(4-\Delta=-4,-6)$ which suggest the possibility of unphysical higher-order poles to be cancelled by corresponding zeroes in the tensors. By inspection, we have found another nonpolynomial form for these tensors which is characterized by scale dimension 6 and rational $Q_{i}^{\omega}$ with simple poles $\sim 1 /\left(q^{2}-2 k^{2}\right), 1 /\left(q^{2}+2 k^{2}\right)$ (higher-order poles and $1 / k^{2}$ poles are absent in this basis). The corresponding amplitudes have again dimension -2 . 
In conclusion, the general $\langle T T T\rangle$ can be put into a form which does not explicit contain higher-order poles and possess a smooth limit $k^{2} \rightarrow 0$ with $q^{2} \neq 0$. This form is convenient in the kinematical region $q^{2}>2 k^{2} \geq 0$; other choices of basis may be relevant for different corners of the phase space, say $q^{2} \rightarrow 0$ with $k^{2} \neq 0$.

\section{Renormalization and scheme-dependence of the amplitudes.}

As discussed at the end of Section 2.3, the form of $\langle T T T\rangle$ in (3.8) can be changed by adding counterterms, which are polynomials of the momenta of maximal dimension four. The counterterms cannot be arbitrary, because they would change the definition of the stress tensor, e.g. by transforming a trace anomaly into a gravitational anomaly; therefore, the counterterms should be already present in the solution of the Ward identities just found. We can identify them as the tensors with $\Delta=4$ and $\delta_{T} \leq 4$, which actually are polynomials; the corresponding dimensionless amplitudes are scheme-dependent by the shifts $A_{\omega} \rightarrow A_{\omega}+$ const.. Such tensors are:

- The null tensor $\mathcal{T}_{8}$ which vanishes identically.

- The tensor $\mathcal{T}_{0+}$ which matches the scheme-dependent $D^{2} \mathcal{R}$ term in the trace anomaly; this verifies the ambiguity $a^{\prime} \rightarrow a^{\prime}+$ const. at the level of the three-point function.

- The tensor $\mathcal{T}_{2+}$, which accounts for the $\mu$-dependence of the logarithms in the amplitude $f_{2+}$ (Eq. 3.15) ); such dependence instead cancels out in the odd combination $f_{2-}$, which multiplies a $\Delta=\delta_{T}=6$ tensor.

- The tensor $\mathcal{T}_{\Lambda}$ coming from the one-point function off criticality.

These results are in agreement with earlier analyses based on the lower-point functions and the effective action [13] [5] [16][21], and provide a check of our derivation.

Note that two further tensors, $\mathcal{T}_{11}$ and $\mathcal{T}_{12}$, have dimensions $\delta_{T}=4$ and $\Delta=6$, so that they cannot mix with counterterms. Here we have checked that their dimension cannot be further lowered by changing the basis: the mixing with other tensors is not actually possible owing to their higher $\delta_{T}$ value.

In conclusion, the $\delta_{T}=6$ tensors in Table 2 (and the previous $\mathcal{T}_{11}$ and $\mathcal{T}_{12}$ ) identify amplitudes $A_{i}\left(q^{2}, k^{2}\right)$ which are finite and scheme-independent with scale dimension $(-2)$. In particular, we found the desired result of matching the (universal) anomaly coefficients $a, c$ with two of these amplitudes, namely $A_{E}$ and $A_{W}$ in (3.9).

\footnotetext{
ब Note that all tensors become polynomial at $k^{2}=0$; the solution of the Ward identities at $k^{2}=0$ will be further discussed in Section 4.
} 


\begin{tabular}{|c|c|c|c|c|c|c|c|c|c|c|c|c|}
\hline & \multicolumn{5}{|c|}{ transverse } & \multicolumn{5}{|c|}{ non-transverse } & \multicolumn{2}{|c|}{ total } \\
\hline$k^{2} \neq 0$ & \multicolumn{3}{|c|}{$\overline{\delta_{T}=6}$} & \multicolumn{2}{|c|}{$\overline{\delta_{T}=4}$} & \multicolumn{2}{|c|}{$\overline{\delta_{T}=6}$} & \multicolumn{3}{|c|}{$\overline{\delta_{T} \leq 4}$} & $\delta_{T}=6$ & $\delta_{T} \leq 4$ \\
\hline Diff & \multicolumn{3}{|c|}{14} & \multicolumn{2}{|c|}{$2(3)$} & \multicolumn{2}{|c|}{2} & \multicolumn{3}{|c|}{3} & 16 & 5 \\
\hline Weyl & 7 & 2 & 5 & $(1)$ & 2 & 1 & 1 & 1 & 1 & 1 & 10 & 2 \\
\hline value & $(t r=0)$ & $a, c$ & 0 & null & 0 & $f_{2-}^{c}$ & 0 & $f_{2+}^{c}$ & $a^{\prime}$ & 0 & & \\
\hline label $i$ & $1, \ldots, 7$ & 9,10 & $13, . ., 17$ & 8 & 11,12 & $2-$ & $0-$ & $2+$ & $0+$ & $\Lambda$ & & \\
\hline$k^{2}=0$ & \multicolumn{3}{|c|}{ 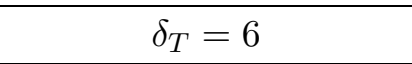 } & \multicolumn{2}{|c|}{$\overline{\delta_{T}=4}$} & \multicolumn{2}{|c|}{ 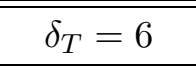 } & \multicolumn{3}{|c|}{ 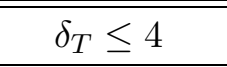 } & $\delta_{T}=6$ & $\delta_{T} \leq 4$ \\
\hline Diff & \multicolumn{3}{|c|}{12} & \multicolumn{2}{|c|}{$4(5)$} & \multicolumn{2}{|l|}{2} & \multicolumn{3}{|c|}{2} & 14 & 6 \\
\hline Weyl & 7 & 2 & 3 & (1) & 4 & 1 & 1 & & & 1 & 10 & 1 \\
\hline value & $(t r=0)$ & $a, c$ & 0 & null & 0 & $f_{2}^{c}\left(q^{2}\right)$ & 0 & & & 0 & & \\
\hline label $i$ & $1, . ., 7$ & 9,10 & $15, \ldots, 17$ & 8 & $11, . ., 14$ & 2 & $0-$ & & & $\Lambda$ & & \\
\hline
\end{tabular}

Table 3: Summary of the properties of the solutions to the Ward identities for $k^{2} \neq 0$ and $k^{2}=0$. Using the notations of Table 2, we report the number of solutions, split by the $\delta_{T}$ value, both off criticality (Diff line) and at criticality (Weyl); we also indicate the critical limit of the amplitudes (value) and their labels.

\section{Limit of the solution for $k^{2} \rightarrow 0$}

As already said, the limit is smooth in our choice of $\mathcal{T}_{\omega}$ basis; however, one tensor becomes a linear combination of the others and should be removed. Actually, for $k^{2}=0$, the two tensor structures of the two-point function $\left\langle\left(h_{1} \cdot T(k)\right)\left(h_{2} \cdot T(-k)\right)\right\rangle$ are degenerate (see Eq.(2.30)), so that the Diff Ward identity (3.2) looses one inhomogeneous solution. Moreover, the amplitude $f_{2}\left(k^{2}\right)$ becomes singular at criticality (see Eq.(2.32)); thus, the corresponding term $f_{2}\left(k^{2}\right)\left[\mathcal{T}_{2+}-\mathcal{T}_{2-}\right]$ is subtracted from $\langle T T T\rangle$ :

$$
\begin{aligned}
& \left\langle\left(h_{1} \cdot T\right)\left(h_{2} \cdot T\right)\left(h_{3} \cdot T\right)\right\rangle_{\mathrm{reg}, k^{2}=0} \\
& \equiv \lim _{k^{2} \rightarrow 0}\left\{\left\langle\left(h_{1} \cdot T\right)\left(h_{2} \cdot T\right)\left(h_{3} \cdot T\right)\right\rangle-\frac{f_{2}\left(k^{2}\right)}{2}\left[\mathcal{T}_{2+}-\mathcal{T}_{2-}\right]\right\} .
\end{aligned}
$$

The $k^{2}=0$ solution thus contain $21=(17$ transverse +4 non-transverse $)$ independent tensors. The analysis of the Ward identities discussed before has been independently repeated at the point $k^{2}=0$ and matched with the result of the limit from $k^{2} \neq 0$. A summary of the properties of the solutions for both $k^{2}=0$ and $k^{2} \neq 0$ is given in Table 5; note that some of the tensors have lower $\delta_{T}$ values at $k^{2}=0$, but the number and type of homogeneous solutions remains the same. 


\subsection{Critical points: conformal isometries of flat space}

In the previous Section, we obtained the general form (3.8) of $\langle T T T\rangle$, which contains 9 scheme-independent amplitudes at criticality, namely $A_{i}, i=1, \ldots, 7, A_{E}$ and $\left(A_{W}, f_{2-}\right)$. Previous approaches in coordinate space have instead found three independent non-contact tensor structures [19] 20]: two parameterized by the $a$ and $c$ anomalies and one traceless transversell. The disagreement is due to the fact that, in our derivation, we did not impose the symmetry under the regular (i.e. global) conformal transformations of flat space, belonging to the group $S O(d+1,1)[17] 15$; as is well known, these symmetries almost completely determine the functional form of the two- and three-point functions in coordinate space. Moreover, we did not considered the functional relations among amplitudes due to the exchange symmetry of $T\left(k_{1}\right)$ with $T\left(k_{3}\right)$.

On the other hand, the disadvantage of the coordinate-space approach is that it yields the expressions of correlators for non-coincident points, which are rather difficult to renormalize [19]. Furthermore, the coordinate approach cannot be easily extended away from criticality because the Diff Ward identities become involved.

In the following, we rederive the Ward identities for the $S O(d+1,1)$ conformal symmetry in flat momentum space, without neglecting the contact terms and consistently following the conventions set in this paper. As is well known [15], a $S O(d+1,1)$ conformal transformation is a combinations of a reparametrization and a Weyl scaling which map the Minkowski metric into itself: the precise notion is that of conformal isometries of the flat metric, which are associated to conformal Killing vectors [23]. A general metric $g^{\mu \nu}(x)$ possesses a conformal isometry if it satisfies:

$$
0=\left(\delta_{\epsilon}+\delta_{\sigma}\right) g^{\mu \nu}(x) \equiv D^{(\mu} \epsilon^{\nu)}(x)-2 \sigma(x) g^{\mu \nu}(x),
$$

where $\epsilon^{\mu}$ is the conformal Killing vector; the associated Weyl scaling $\sigma(x)=(D \cdot \epsilon) / d$ is found by taking the trace of (3.19). In flat space, the solutions of (3.19) generate the $S O(d+1,1)$ conformal group [17]:

$$
\epsilon^{\mu}(x)=b^{\mu}+\lambda x^{\mu}+\omega_{\nu}^{\mu} x^{\nu}+a^{\mu} x^{2}-2(a \cdot x) x^{\mu}, \quad(d>2) .
$$

We recall that $\delta_{\epsilon}=-\mathcal{L}_{\epsilon}$ is (minus) a Lie derivative and that the combination $\left(\delta_{\epsilon}+\delta_{\sigma}\right)$ is still covariant because the Weyl transformation is local. The usual conformal Ward identities in flat space 15] are nothing else than the covariance conditions for the fields. For example, a scalar and a vector field satisfy:

$$
0=-\left(\delta_{\epsilon}+\delta_{\sigma}\right) \varphi=(\epsilon \cdot \partial) \varphi+\frac{\Delta}{d}(\partial \cdot \epsilon) \varphi,
$$

|| The three independent structures were originally found in free field theory by the first of Refs. 19, and were confirmed by the subsequent analyses of the conformal symmetry. 


$$
0=-\left(\delta_{\epsilon}+\delta_{\sigma}\right) A_{\mu}=(\epsilon \cdot \partial) A_{\mu}+\left(\partial_{\mu} \epsilon^{\alpha}\right) A_{\alpha}+\frac{\Delta-1}{d}(\partial \cdot \epsilon) A_{\mu} .
$$

The field $\varphi, A_{\mu}$ are said quasi-primary, because they transform homogeneously under Weyl with scaling dimension $\Delta$. In the case of the stress tensor, we must pay attention to the effects of the trace anomaly, and the naive (classical) formulas (3.21) should not be taken for granted.

We reconsider the Ward identities 2.20) for Diff and Weyl transformations of the generating functional and specialize them for a conformal isometry (3.19) (for more details, see Appendix B). The two identities can be combined to obtain the sum of three terms: respectively, the stress-tensor $(n+1)$-function times $\left(\delta_{\epsilon}+\delta_{\sigma}\right) g$, the $n$-th point function and the $n$-th variation of the anomaly. Since the first term vanishes for conformal isometries, this Ward identity closes on the $n$-point functions and yields a finite set of differential equations with inhomogeneous term given by the anomaly. These equations put additional constrains on the solutions of the two separate Ward identities, which apply to general metrics without conformal isometries.

The conformal Ward identity for the two-point function is:

$$
\begin{aligned}
& -\left[\left(\delta_{\epsilon}+\delta_{\sigma}\right)_{1}+\left(\delta_{\epsilon}+\delta_{\sigma}\right)_{2}\right]\left\langle T_{\alpha \alpha^{\prime}}\left(x_{1}\right) T_{\beta \beta^{\prime}}\left(x_{2}\right)\right\rangle \\
& =-\left.2 \frac{\delta^{2}}{\delta g^{\alpha \alpha^{\prime}}\left(x_{1}\right) \delta g^{\beta \beta^{\prime}}\left(x_{2}\right)} \int \sqrt{g} \sigma \mathcal{A}(g)\right|_{\sigma=(\partial \cdot \epsilon) / 4},
\end{aligned}
$$

where the metric variations are evaluated in flat space. The conformal transformation of the stress tensor reads [15]:

$$
-\left(\delta_{\epsilon}+\delta_{\sigma}\right) T_{\alpha \beta}=\left[(\epsilon \cdot \partial)+\frac{d-2}{d}(\partial \cdot \epsilon)\right] T_{\alpha \beta}+\left(\partial_{(\alpha} \epsilon^{\nu}\right) T_{\nu \beta)} .
$$

For scale variations $\left(\epsilon^{\mu}=b x^{\mu}\right)$ and in four-dimensional momentum space, Eq. 3.22) becomes:

$$
\left(p \cdot \partial_{p}-4\right)\left\langle\left(h_{1} \cdot T(p)\right)\left(h_{2} \cdot T(-p)\right)\right\rangle=2 \mathcal{A}\left(p, h_{1} ;-p, h_{2}\right),
$$

where the r.h.s. is the second variation of the integrated trace anomaly (1.1) in the notation introduced before (see Eq.(3.7)). This variation only contains the Weyl term, because the Euler term is topological invariant and $\sqrt{g} D^{2} \mathcal{R}$ is a total derivative; therefore, the r.h.s. of (3.24) is a traceless-transverse tensor. By plugging in the explicit expressions of the anomaly (3.7) and of $\langle T T\rangle$ (2.30), one verifies that the coefficient of the logarithm in spin-two amplitude $f_{2}\left(p^{2}\right)$ is equal to the anomaly number $c$, as anticipated in Eq.(2.32) [13] [5].

For special conformal transformations, parameterized by $a_{\mu}$ in (3.20), the Ward identity (3.22) becomes a second-order differential equation in momentum space; we do not 
solve it but rely on earlier studies in coordinate space (see e.g. Ref. [5]) to conclude that it is automatically satisfied by the critical form of $\langle T T\rangle$ given by (2.30) with $f_{0}^{c}, f_{2}^{c}$ in (2.31,2.32).

In conclusion, we have found that the conformal Ward identities for the stress-tensor $n$-point correlators contain an inhomogeneous term given by the $n$-th variation of the trace anomaly, and that they amount to a set of differential equations for the scalar amplitudes at criticality. At the three-point level, the equations for special conformal transformations, together with the functional relations for the $(1 \leftrightarrow 3)$ symmetry, presumably determine the form of the traceless transverse amplitudes, $A_{i}^{c}\left(q^{2}, k^{2}\right), i=1, \ldots, 7$, leading to an agreement with the coordinate-space result 19] 20]. We shall not carry out this analysis, because we are more interested in off-critical behavior of the traceful terms in $\langle T T T\rangle$, which describe the renormalization-group flow of the anomaly coefficients $a$ and $c$.

\section{Trace anomaly: reduction formulae and sum rules}

\subsection{Reduction formulae}

We now describe the inversion of the $\langle T T T\rangle$ expansion (3.8) which extracts the amplitudes $A_{\omega}\left(q^{2}, k^{2}\right)$ from an explicit expression of the three-point function, such as the result of a calculation. The latter can be expanded in the canonical basis of Bose-symmetric tensors $\mathcal{P}_{i}, i=1, \ldots, 77$ (see Table 1), leading to a set of know coefficient functions $u_{i}\left(q^{2}, k^{2}\right)$. We then write (see also (3.17)):

$$
\begin{aligned}
\langle T T T\rangle & =\sum_{\omega=1}^{22} A_{\omega} \mathcal{T}_{\omega}=\sum_{i=1}^{77} \sum_{\omega=1}^{22} A_{\omega}\left(q^{2}, k^{2}\right) Q_{i}^{\omega}\left(q^{2}, k^{2}\right) \mathcal{P}_{i}\left(k_{1}, h_{1}, k_{2}, h_{2}, h_{3}\right) \\
& =\sum_{i=1}^{77} u_{i}\left(q^{2}, k^{2}\right) \mathcal{P}_{i} .
\end{aligned}
$$

After the elimination of the null vector, the $21 \mathcal{T}_{\omega}$ 's and the $76 \mathcal{P}_{i}$ 's form two sets of linearly-independent "vectors"; therefore, Equation (4.1) defines the linear problem of finding the "coefficients" $A_{\omega}$ by inverting the $21 x 76$ matrix $Q_{i}^{\omega}$ of maximal rank. The solution is unique and expresses the $21 A_{\omega}$ 's as linear combinations of the $u_{i}$ 's, together with 55 linear relations among the $u_{i}$ 's.

These constraints verify that the calculation, besides being correct, satisfies the Diff Ward identities in our specific formulation, which includes a complete choice of renormalization scheme and of stress-tensor definitions. This leads to the practical problem that a given calculation may not immediately fit our scheme, and thus the check of our Ward identities (i.e. of the 55 constraints) may require modifications of the calculation rules and 
become a painful process. For example, in Section 5 it is shown that a Feynman-graph calculation of $\langle T T T\rangle$ needs the inclusion of $T_{\mu \nu}$ vertices with an increasing number of legs in order to match our definition of the stress tensor by differentiation of the generating functional, Eq.(2.19). There is basically no short cut to this problem if one really needs to determine the whole expression of $\langle T T T\rangle$ including the scheme-dependent terms.

An important simplification occurs in deriving the scheme-independent $\delta_{T}=6$ amplitudes: firstly, one can limit the inversion (4.1) to the 15-dimensional subset of $\delta_{T}=6$ basic vectors $\mathcal{P}_{i}, i=1, \ldots, 15$ (see Table 11); secondly, these amplitudes are independent of renormalization scheme and $T_{\mu \nu}$ definition用, so that eventual constraints among the $u_{i}$ 's, should be automatically verified in any calculation $\square$. More precisely, for $k^{2} \neq 0$ the number of amplitudes is $14+2$ (transverse+non-transverse) (see Table 3): the two nontransverse tensors are not linearly independent in the $\delta_{T}=6$ basis but otherwise known, thus there are 15 unknowns and no constraints. For $k^{2}=0$, there are $12+2$ unknown amplitudes (the inhomogeneous ones are still dependent) and 2 constraints; finally, at the critical point there are $9+1$ unknowns and 5 constraints in both cases.

Among the $\delta_{T}=6$ amplitudes, we are particularly interested to $A_{E}$ and $A_{W}$, which reduce to the anomaly coefficients $a, c$ at criticality (see Section 3.1). Hereafter, we present the determination of the $\delta_{T}=6$ amplitudes at the point $k^{2}=0$, which is sufficient for our later purposes (the general case can be found in the Mathematica notebook associated to this paper). The $12+1$ independent $k^{2}=0$ amplitudes are obtained by inverting the (numeric) $13 \times 15$ matrix $Q_{i}^{\omega}\left(q^{2}, k^{2}=0\right)$ of Eq. (4.1) restricted to the subspace of $\left(\delta_{T}=6\right)$ $\mathcal{P}_{i}$ 's. We remark that this linear problem can be equivalently solved by introducing a dual basis of tensors $\mathcal{T}_{\omega}^{*},\left(\mathcal{T}_{\omega}^{*}, \mathcal{T}_{\omega^{\prime}}\right)=\delta_{\omega, \omega^{\prime}}$, where the brackets denote a suitable degenerate scalar product which vanishes on the $\delta_{T}<6$ basis [2].

We finally obtain the following pair of reduction formulae for the anomaly amplitudes $\left(k^{2}=0\right)$ :

$$
\begin{aligned}
A_{E}\left(q^{2}, 0\right) & =\frac{1}{36}\left(4 u_{2}-4 u_{4}-u_{5}+2 u_{8}-2 u_{11}+12 u_{14}+3 u_{15}\right) \\
A_{W}\left(q^{2}, 0\right) & =\frac{1}{108}\left(-8 u_{2}+8 u_{4}+2 u_{5}-4 u_{8}+4 u_{11}+12 u_{14}+3 u_{15}\right)
\end{aligned}
$$

together with the two constraints:

$$
u_{1}=\frac{1}{2} u_{3}, \quad u_{12}=0
$$

Note that the formulae 4.2, 4.3) are invariant under the changes of basis, $\mathcal{T}_{\omega} \rightarrow \mathcal{T}_{\omega}+$ $\sum_{i=1}^{7} r_{i} \mathcal{T}_{i}, \omega=E, W$, which add traceless-transverse terms to the anomaly tensors.

** An argument for the definition-independence is given in Section 5 .

${ }^{\dagger \dagger}$ Note that the null vector has $\delta_{T}=4$ and is automatically excluded from this basis. 
Another interesting reduction formula yields a combination of the $\langle T T\rangle$ amplitudes which is associated to a $\delta_{T}=6\langle T T T\rangle$ tensor (see Table 3):

$$
\frac{1}{2 q^{2}}\left(2 f_{2}\left(q^{2}\right)+f_{0}\left(q^{2}\right)-f_{0}\left(k^{2}=0\right)\right)=\frac{u_{3}}{4} ;
$$

this result gives a useful check. The complete set of 13 reduction formulae is reported in Appendix D.

At the critical point, the number of independent tensor reduces to 10 and there are 5 constraints; in this case, the reduction formulae determine the anomaly coefficients (see Eq. (3.9)):

$$
\begin{aligned}
& a=\frac{q^{2}}{12 \lambda}\left(-u_{11}-u_{13}+4 u_{14}+u_{15}\right) \\
& c=\frac{q^{2}}{36 \lambda}\left(2 u_{11}+2 u_{13}+4 u_{14}+u_{15}\right) .
\end{aligned}
$$

The constraints at criticality are:

$$
\begin{aligned}
u_{1} & =\frac{1}{2} u_{3}, \quad u_{12}=0, \\
u_{2} & =\frac{1}{4}\left(4 u_{4}+u_{5}-2 u_{8}-u_{11}-3 u_{13}\right), \\
u_{3} & =\frac{1}{9}\left(9 u_{6}-2 u_{11}-2 u_{13}-4 u_{14}-u_{15}\right), \\
u_{9} & =\frac{1}{4}\left(-u_{10}+u_{11}+u_{13}\right) .
\end{aligned}
$$

Equations (4.6,4.7) allow to compute the trace anomalies by standard flat-space perturbative calculations, and can be useful for interacting theories.

Infrared divergences. As emphasized, the amplitudes $u_{i}\left(q^{2}, k^{2}=0\right), i=1, \ldots, 15$ are ultraviolet finite and satisfy un-subtracted dispersion relations of the type (2.14)) away from critical points. However, some amplitudes may develop infrared singularities at criticality, which are introduced by the kinematical limit $k^{2}=0$. This fact is illustrated by the formula (4.5) (see also Table 3). For $k^{2} \neq 0$, the $\left(\delta_{T}=6\right)$ tensor $\mathcal{T}_{2}$ - would identify a finite amplitude with safe critical limit $m \rightarrow 0$, namely $f_{2-}^{c} / q^{2} \sim \log \left(q^{2} / k^{2}\right) / q^{2}$. For $k^{2}=0$, the critical limit is instead $f_{2}^{c}\left(q^{2}\right) / q^{2} \sim \log \left(q^{2} / m^{2}\right) / q^{2}$, with the physical mass $m$ acting as infrared regulator (rather then the renormalization scale $\mu$ controlling the ultra-violet singularity of the $\langle T T\rangle$ amplitude (2.32)).

The standard solution to this problem amounts to the choice of another point $k^{2} \neq 0$ for the reduction formulae at criticality. However, this is not necessary for the derivation of the anomaly amplitudes Eqs.(4.2, 4.3, 4.6, 4.7), which are free of infrared singularities. Actually, these amplitudes are related to the trace of the stress tensor, which vanishes as an operator at criticality due to Weyl invariance; thus, these amplitudes contain the anomalous pole only. 


\subsection{Sum rules for the renormalization-group flow of $a$ and $c$}

We start by recalling the sum rule for the two-dimensional central charge $c$, Eq. (1.2) [18]: we present a simplified version of the dispersive proof of Ref. [5], which can be conveniently generalized to four dimension. In Section 2.3, we wrote the general form of $\langle T T\rangle$ satisfying the Diff Ward identity, Eq.(2.30); in two dimensions, this reduces to:

$$
\left\langle\left(h_{1} \cdot T(p)\right)\left(h_{2} \cdot T(-p)\right)\right\rangle=f\left(p^{2}\right)\left(p^{2}\left(h_{1}\right)-\left(p \cdot h_{1} \cdot p\right)\right)\left(p^{2}\left(h_{2}\right)-\left(p \cdot h_{2} \cdot p\right)\right) .
$$

The spin-zero scalar amplitude $f\left(p^{2}\right)$ has dimension $(-2)$ and is finite and scheme-independent, namely its renormalization is analogous to that of the four-dimensional amplitudes $A_{3}\left(q^{2}, k^{2}\right)$ of $\langle A V V\rangle$ (Section 2.1) and $A_{E}, A_{W}$ of $\langle T T T\rangle$; note, however, the difference in the singlevariable dependence. The critical limit is:

$$
f\left(p^{2}\right) \rightarrow-\frac{c \pi}{3} \frac{1}{p^{2}}, \quad \text { (critical points), }
$$

where $c$ is the Virasoro central charge and $T$ has the standard two-dimensional normalization [5]. The amplitude satisfies the un-subtracted dispersion relation:

$$
\begin{aligned}
f\left(p^{2}\right) & =\int \frac{d s}{\pi} \frac{\operatorname{Im} f(s)}{s-p^{2}}, \\
\operatorname{Im} f(s) & =\frac{\pi^{2}}{3} c(s)
\end{aligned}
$$

which also identifies $\operatorname{Im} f(s)$ with the spectral measure $c(s)$ introduced in (1.2). Its critical limit is:

$$
c(s) \rightarrow c \delta(s), \quad \text { (critical points) }
$$

We now introduce the following sum rule off-criticality,

$$
\Sigma\left(m^{2}\right)=\frac{3}{\pi^{2}} \int_{0}^{\infty} d s \operatorname{Im} f\left(s, m^{2}\right)=\int_{0}^{\infty} d s c\left(s, m^{2}\right)
$$

and explicitly write the dependence on the mass scale $m$ introduced by the relevant (renormalized) coupling constant $g$. In the following, we would like to prove that $\Sigma\left(m^{2}\right)$ is actually independent of $m$,

$$
\Sigma\left(m^{2}\right)=\Sigma(0), \quad \text { for } 0 \leq m^{2}<\infty
$$

From Eq.(4.13), we know that $\Sigma(0)=c_{U V}$, the central charge of the ultra-violet fixed point. If $\Sigma$ is independent of $m$, it is also renormalization-group invariant, because $t \sim$ $\log (m)$ is the parameter of the renormalization-group flow between the fixed points (UV) and (IR). 
Equation (4.15) can be derived from the general property that the (trace) anomaly is at most a constant; this was shown in the case of $\langle A V V\rangle$ by using dispersion relations (see Eqs.(2.10 2.17)): since the imaginary parts of amplitudes satisfy non-anomalous Weyl Ward identities, the anomaly should be accounted for by the subtraction constants. However, the precise formulation of this argument for $\langle T T\rangle$ (and later on for $\langle T T T\rangle$ ) requires the dispersive analysis of all the amplitudes related by the Ward identity; we prefer to use another argument based on the dimensional analysis and the renormalization group, which is presented hereafter.

The amplitude $f\left(p^{2}, m^{2}\right)$ in (4.9) is finite and scheme-independent: there is no wavefunction renormalization because the stress-tensor has no anomalous dimension, while the coupling-constant renormalization makes the quantity finite and brings in the scale $m$. Infrared divergences are also excluded for this quantity in two dimensions [5]. The imaginary part of $f\left(p^{2}, m^{2}\right)$ has support on the real positive $s$-axis and goes to infinity as $o(1 / s)$, i.e. faster than the behavior allowed by its scale dimension, such that the integral in (4.14) is finite. This extra convergence can be verified perturbatively close to the UV fixed point [1][5]: since $f\left(p^{2}, m^{2}\right)$ measures the trace anomaly, it corresponds to an expectation value involving one $\Theta$ operator (at least); we thus can write,

$$
\begin{array}{rlrl}
f\left(p^{2}, m^{2}\right) & =\langle\Theta(p) \cdots\rangle=\beta(g)\langle\phi(p) \cdots\rangle & \\
& \sim y g m^{y} s^{-1-y / 2}, \quad \text { for } s \rightarrow \infty
\end{array}
$$

where $y>0$ is the dimension of the coupling constant $\left(g_{o} \equiv g m^{y}\right)$, and we used the relation $\Theta=\beta(g) \phi$ expressing the trace off-criticality as the beta function times the perturbing field [28].

In conclusion, $\Sigma\left(m^{2}\right)$ (4.14) does not contain any regulator for the integrand or the integral; it depends on a single dimensionful parameter, i.e. $m$, and thus is necessarily constant (and renormalization-group invariant).

The sum rule (4.14) implies some conditions on its integrand $c(s)$; we can write, in general [5]:

$$
c(s)=c_{0} \delta(s)+c_{1}\left(s, m^{2}\right) .
$$

The function $c_{1}\left(s, m^{2}\right)$ should be a representation of the delta-function with spreading parameterized by $m^{2}$, so that the ultra-violet limit $m \rightarrow 0$ matches the critical form (4.13). In the opposite limit $m \rightarrow \infty, c_{1}\left(s, m^{2}\right)$ becomes very broad and it contributes infinitesimally to the correlator $\langle T T\rangle$ for any fixed scale $s=s_{0}$; therefore, $c(s)$ must be redefined in the infrared scale-invariant theory $(m=\infty)$ by means of the weak limit:

$$
c(s) \rightarrow c_{0} \delta(s), \quad c_{0}=c_{I R}, \quad(m=\infty)
$$


This Equation identifies $c_{0}$ with the infra-red value of the central charge $\left(c_{0}\right.$ is another renormalization-group invariant). We can finally rewrite the sum rule (4.14) in the more familiar form (1.2) [18]:

$$
c_{U V}=\int_{0}^{\epsilon} d s c(s)+\int_{\epsilon}^{\infty} d s c(s)=c_{I R}+\int_{\epsilon}^{\infty} d s c_{1}\left(s, m^{2}\right) .
$$

This equation implies the $c$-theorem in the integrated form, $c_{U V}>c_{I R}$, because the spectral measure $c(s)$ is positive definite [18]. Furthermore, the integral of $c(s)$ up to a fixed (finite) scale defines a function $c(g)$ off-criticality [5], which interpolates between $c_{U V}$ and $c_{I R}$ and satisfies the $c$-theorem in the differential form [1]: $d c(g) / d \log (m) \equiv$ $-\beta(g) d c(g) / d g<0$.

The four dimensional sum rules for the anomaly coefficients $a$ and $c$ are obtained by similar arguments. The corresponding scalar amplitudes $A_{\omega}\left(q^{2}, k^{2}=0\right), \omega=E, W$, are extracted from the three-point function $\langle T T T\rangle$ by the procedure explained in the previous Section; this also applies to the imaginary parts w.r.t. $q^{2}$ because the tensors $\mathcal{T}_{\omega}$ are polynomial at $k^{2}=0$ :

$$
\operatorname{Im}\langle T T T\rangle=\operatorname{Im} A_{E}(s, 0) \mathcal{T}_{E}+\operatorname{Im} A_{W}(s, 0) \mathcal{T}_{W}+\cdots, \quad\left(k^{2}=0\right) .
$$

The amplitudes have dimension $(-2)$ and satisfy un-subtracted dispersion relations; their critical limits (3.9) imply:

$$
\begin{aligned}
\operatorname{Im} A_{E}(s, 0) & \rightarrow-\pi \lambda a \delta(s), \\
\operatorname{Im} A_{W}(s, 0) & \rightarrow-\pi \lambda c \delta(s), \quad \text { (critical points). }
\end{aligned}
$$

The sum rule for the $a$ anomaly is defined in analogy with (4.14):

$$
\Sigma_{E}\left(m^{2}, 0\right)=-\frac{1}{\pi \lambda} \int_{0}^{\infty} d s \operatorname{Im} A_{E}\left(s, k^{2}=0, m^{2}\right)
$$

with the $m$-dependence explicitly written. This integral is meant to extend over the support of $\operatorname{Im} A_{E}$.

We now follow the same steps as for the two-dimensional case, Eqs.(4.15-4.19): we first argue that $\Sigma_{E}$ is $m$-independent and equal to the ultra-violet value $a_{U V}$, provided that it is finite and does not involve any regulator. The integrand is indeed finite and schemedependent, as already stressed; the integral is convergent if the stress tensor is "improved" at both the UV and IR fixed points, i.e. $\Theta \equiv 0$ at criticality (apart from anomalies). Actually, $\operatorname{Im} A_{E}$ is related to an expectation value involving the $\Theta$ operator, whose vanishing at the UV (IR) critical point forces the asymptotic behavior of $\operatorname{Im} A_{E}\left(s, 0, m^{2}\right)$ to be faster than $1 / s$, the scale-invariant law, for $s \rightarrow \infty$ (respectively, $s \rightarrow 0$ ). 
The improvement is implied by Weyl invariance, assumed throughout this paper, but is not guaranteed for a generic fixed point: as already said, four-dimensional scale invariance only require $\Theta$ to be a derivative operator. Near a generic UV fixed point, it is rather natural to assume the improvement and the relation $\Theta=\beta(g) \phi$, Eq.(4.16) [28]. Near the IR point, it is less obvious: for example, the spontaneous symmetry breaking of a global symmetry may lead to an infrared critical theory without improved stress tensor [5]. We are aware that the hypothesis of improvement at the IR fixed point (convergence of the sum rule) may exclude interesting non-perturbative renormalization-group flows from our analysis.

The constancy of the sum rule (4.22) again constrains the amplitude to be of the form (4.17):

$$
-\frac{1}{\pi \lambda} \operatorname{Im} A_{E}\left(s, k^{2}=0, m^{2}\right)=a_{0} \delta(s)+a_{1}\left(s, m^{2}\right) ;
$$

where the smooth function $a_{1}$ is a representation of the delta function for $m \rightarrow 0$, and the explicit flow-invariant delta term survives in the infrared theory. Note that the scaleinvariant power law, $\operatorname{Im} A_{E}(s, 0,0) d s \sim d s / s$, is excluded at criticality because it would imply a non-vanishing $\Theta$ operator and a divergent sum rule, as emphasized.

We finally obtain the sum rule for the four-dimensional anomaly, analog of Eq. (1.2):

$$
a_{U V}-a_{I R}=-\frac{1}{\pi \lambda} \int_{\epsilon}^{\infty} d s \operatorname{Im} A_{E}\left(s, 0, m^{2}\right)
$$

The other four-dimensional anomaly number $c$ satisfies a similar sum rule involving $\operatorname{Im} A_{W}\left(s, 0, m^{2}\right)$. Equation (4.24) is the announced result of this Section, which is followed by a number of remarks:

- If $\operatorname{Im} A_{E}\left(s, 0, m^{2}\right)$ were positive definite, then Eq.(4.24) would prove the $c$-theorem in four dimensions, $a_{U V}>a_{I R}$, as suggested by the non-trivial examples mentioned in the Introduction [6] [7]. We do not discuss the positivity in this paper, but only add some remarks in the Conclusions.

- On the other hand, the corresponding result for the Weyl-squared term, $c_{U V}>c_{I R}$, is not true in general, as shown by known counter-examples [5] [6]; the respective amplitude $\operatorname{Im} A_{W}\left(s, 0, m^{2}\right)$ cannot be positive definite in general, although its critical limit is always positive, i.e. $c_{U V}, c_{I R}>0$, due to the positivity of $\langle T(x) T(0)\rangle$ at criticality $(|x| \neq 0)[5]$. These facts suggest the absence of naive back-of-the-envelope positivity arguments.

- The other three $\delta_{T}=6$ amplitudes $A_{i}$ corresponding to traceful tensors $\mathcal{T}_{i}(i=$ $15, \ldots, 17$ in Table 3) also satisfy analogous sum rules; however, their vanishing 
critical limit imply null sum rules 4.22), $\Sigma_{i}=0$, for $i=15, \ldots, 17$ : thus, the imaginary parts of these amplitudes cannot have definite sign.

- We stress that the positivity of $\operatorname{Im} A_{E}\left(s, 0, m^{2}\right)$ is not necessary for the derivation of the sum rule (4.24); its smooth part $a_{1}\left(s, m^{2}\right)$ can be any representation of the delta function which becomes very broad in the infrared limit $m \rightarrow \infty$ (this is assured by the decoupling of massive states).

We conclude this section with a remark on the chiral anomaly, analysed in Section 2.1: the sum rule (4.24) can also be written for the flow of the chiral anomaly, as is clear from Eq.(2.17), analogous to (4.22). However, there is an important difference: the role of the Weyl symmetry is now played by the chiral symmetry, such that "critical theory" should be replaced with "theory with massless fermions". These are not equivalent properties, because it is conceivable a renormalization-group flow with chiral symmetry, for example a QCD-like theory with massless quarks. In this case, the relevant operator causing the flow yields the stress-tensor trace according to (4.16), but does not couple to the chiral current; as a consequence, $\operatorname{Im} A_{3}(s, 0)$ in (2.17) remains equal to its UV critical form of a delta function and there is no actual flow of the chiral anomaly [22]:

$$
C_{U V}=C_{I R}, \quad \text { (RG flows in chiral theories). }
$$

Furthermore, the term $\operatorname{Im}\langle A V V\rangle \sim \delta(s)$ means that the chiral anomaly is saturated by massless on-shell intermediate states $\left(q^{2}=k^{2}=0\right)$ [30]; thus, it can be perturbatively computed either from the fundamental (UV) or the composite (IR) massless fermions; Equation (4.25) corresponds to the 't Hooft anomaly matching conditions [22]. In conclusion, the sum rules for the conformal and chiral anomalies are mathematically identical but may lead to rather different physical consequences.

\section{Test calculations in free field theories}

We now apply the results of Section 4 to the cases of the free massive scalar and Dirac fermion theoriesf; we (re)compute here the anomaly coefficients $a, c$, verify the related sum-rules and check other properties of the reduction formulae.

The stress-tensors three-point correlator is obtained, according Eq.(2.19), by triple variation w.r.t $g^{\mu \nu}$ of the generating functional:

$$
W(g) \equiv \log \int[d \phi] e^{I(\phi ; g)} .
$$

\footnotetext{
${ }^{\dagger}$ Background material for these calculations can be found e.g. in Ref. 5 .
} 
After variations and flat limit one schematically obtains:

$$
\left\langle T_{1} T_{2} T_{3}\right\rangle=\left\langle\delta_{1} I \delta_{2} I \delta_{2} I+\left(\delta_{1} I \delta_{2} \delta_{3} I+\text { perms. }\right)+\delta_{1} \delta_{2} \delta_{3} I\right\rangle+\cdots,
$$

where we denoted by perms. the permutations of labels and we left unspecified some disconnected terms, all containing a $\langle\delta I\rangle$ factor. In terms of Feynman diagrams, the first three contributions to $\langle T T T\rangle$ in Eq. (5.2) are described respectively by three one graviton vertices simply linked in a triangle, by a two graviton vertex doubly linked to a one graviton vertex, and a tadpole over a three graviton vertex.

It is clear that a complete calculation of all contributions - even in a free field theory - is a nontrivial algebraic task. Nevertheless, it should be reminded that the results of Section 4 only involve the subset of $\langle T T T\rangle$ terms that have tensorial dimension $\delta_{T}=6$. We now remark the following simple property: if the action $I$ contains at most two derivatives, any insertions of (variations of) $I$ in the expectation value can raise the tensorial dimension by two at most. It then follows that the second and third contributions to $\langle T T T\rangle$ have tensorial dimension $\delta_{T}<6$ and should not be considered for our purposes. Moreover, owing to the fact that $\left\langle\delta_{g^{\mu \nu}} I\right\rangle \propto \delta_{\mu \nu}$ in a flat space, the leftover disconnected terms in (5.2) also have $\delta_{T}<6$.

In conclusion, we are left with the triangle diagram whose explicit contribution is,

$$
\begin{gathered}
\langle T T T\rangle_{\text {triangle }}=\int \frac{d^{4} L}{(2 \pi)^{4}} V_{h_{3}}(L-q,-L) S(L) V_{h_{1}}\left(L, k_{1}-L\right) S\left(L-k_{1}\right) \\
\times V_{h_{2}}\left(L-k_{1}, q-L\right) S(L-q)
\end{gathered}
$$

where the vertex $V\left(k_{1}, k_{1}\right)$ and the propagator $S(p)$ can be found, e.g. in Ref. [5] (see Equations (3.27-3.30) there).

The imaginary part w.r.t. $q^{2}$ of $\langle T T T\rangle_{\text {triangle }}$ for $q^{2}>0$ and $k^{2}=0$ is obtained by the standard Cutkowsky rules, cutting the lines linked to the vertex $T_{3}$ (the remaining integrals are elementary). After collecting from the resulting expression the coefficients $u_{i}\left(q^{2}, 0\right)$ of the expansion in the canonical $\left(\delta_{T}=6\right)$ basis $\mathcal{P}_{i}, i=1, \ldots 15$, we have tested the formulas of Section 4. We have found that:

- The constraints (4.4) and (4.8) are satisfied off and at criticality, respectively.

- The reduction formulae (4.2,4.3) determine the following expressions for the Euler and Weyl anomaly amplitudes, in the massive scalar theory:

$$
\begin{aligned}
& -\frac{1}{\lambda \pi} \operatorname{Im} A_{E}\left(s, 0, m^{2}\right) \\
& =\theta(x-1) \frac{5}{96 m^{2} x^{4}}\left[32 r x(1+3 x)+\left(-27+80 x+12 x^{2}\right) \log \left(\frac{1-r}{1+r}\right)\right],
\end{aligned}
$$




$$
\begin{aligned}
& -\frac{1}{\lambda \pi} \operatorname{Im} A_{W}\left(s, 0, m^{2}\right) \\
& =-\theta(x-1) \frac{5}{144 m^{2} x^{4}}\left[8 r x(13+3 x)+\left(27+26 x+12 x^{2}\right) \log \left(\frac{1-r}{1+r}\right)\right],
\end{aligned}
$$

with

$$
x=\frac{s}{4 m^{2}}, \quad r=\sqrt{1-\frac{4 m^{2}}{s}} .
$$

In the massive fermion theory, we find:

$$
\begin{aligned}
& -\frac{1}{\lambda \pi} \operatorname{Im} A_{E}\left(s, 0, m^{2}\right) \\
& =-\theta(x-1) \frac{5}{24 m^{2} x^{4}}\left[8 r x(4+17 x)+3\left(-9+25 x+12 x^{2}\right) \log \left(\frac{1-r}{1+r}\right)\right], \\
& -\frac{1}{\lambda \pi} \operatorname{Im} A_{W}\left(s, 0, m^{2}\right) \\
& =\theta(x-1) \frac{5}{72 m^{2} x^{4}}\left[4 r x(52+5 x)+3\left(18+23 x-3 x^{2}\right) \log \left(\frac{1-r}{1+r}\right)\right] .
\end{aligned}
$$

- The sum rules (4.24) for these imaginary amplitudes verify the expected renormalizationgroup flows of the trace anomalies (see e.g. Ref.[5] for the well-known free-field anomalies):

$$
\begin{array}{llc}
a_{U V}-a_{I R}=1, & c_{U V}-c_{I R}=1, & \text { (scalar); } \\
a_{U V}-a_{I R}=11, & c_{U V}-c_{I R}=6, & \text { (Dirac fermion). }
\end{array}
$$

- The amplitudes $A_{i}, i=15,16,17$, of the traceful tensors vanishing at criticality have also been computed (using the formulas in Appendix D); the corresponding sum rules (4.22) are convergent and equal to zero, as expected:

$$
\Sigma_{i}=0, \quad i=15,16,17 .
$$

- The imaginary parts of the Euler and Weyl amplitudes turn out to be positive definite in all the above examples but one, i.e. the bosonic $\operatorname{Im} A_{E}(5.4)$; nevertheless, a positive definite function can be obtained by adding to $\operatorname{Im} A_{E}$ a suitable linear combination of the three non-definite amplitudes $\operatorname{Im} A_{i}, i=15,16,17$, which have vanishing sum rule.

- Infrared divergences occur at criticality for some of the amplitudes $A_{i}, i=1, \ldots, 7$, of the traceless tensors, in agreement with the discussion in Section 4.1; moreover, the formula $f_{2}\left(q^{2}\right) / q^{2} \sim \log \left(q^{2} / m^{2}\right) / q^{2}$ is checked for $m \rightarrow 0$ (including the numerical coefficient computing $c$ again). 
We finally remark that another possible test of the reduction formulae at criticality (4.6, 4.7,4.8) is provided by the free massless vector field; however, the theory becomes interacting off-criticality (there is no flow into the massive free vector), so that the sum rules can only be checked perturbatively. We postpone this analysis to further developments of our work.

\section{Conclusions}

In this paper, we have found the general four-dimensional form of the stress-tensor threepoint function, written as an expansion over scalar amplitudes times tensor structures solution of the Ward identities. We thus made explicit the conditions imposed by the mandatory symmetries, leaving out any dynamical hypothesis. From this result, we have identified the amplitudes corresponding to the two universal trace-anomaly numbers $a$ and $c$, and derived the renormalization-group sum rules which they satisfy.

The main motivation for this analysis has been the extension of the Zamolodchikov $c$ theorem to four dimensions: we did not provide a proof but we tried to clear the ground for further attempts to it. One possible road could be the proof of positivity for the imaginary amplitude entering the $a$ sum rule (4.24) (possibly using the weak-energy condition of Ref.[20]).

Other arguments which stands on specific dynamical hypotheses can hopefully take advantage of our reduced set of variables. For example, exploiting the conditions of $\mathcal{N}=1$ supersymmetry, which is present in most of the non-trivial tests of the would-be $a$-theorem [6] [0]. It would also be interesting to reformulate the dynamical assumptions of Refs. 10 at the level of three-point functions. Finally, the analysis of manifestly positive-definite four-point amplitudes is feasible with the technology presented in this paper.

\section{Acknowledgements}

The authors thank the hospitality of each other authors' Institution at various stages of this collaboration. They also thank D. Anselmi, D. Atlani, C. M. Becchi, J. Bros and Y. Stanev for useful discussions. This work was supported in part by the European Community Network grant FMRX-CT96-0012.

\section{A Notations and conventions}

The four-dimensional trace anomaly is written:

$$
g^{\mu \nu}\left\langle T_{\mu \nu}\right\rangle \equiv\langle\Theta\rangle=\lambda\left(a E-3 c W+a^{\prime} D^{2} \mathcal{R}+r \mathcal{R}^{2}\right), \quad \lambda \equiv \frac{1}{2880 \cdot 4 \pi^{2}} .
$$


where the Euler density and the square of the Weyl tensor are, respectively,

$$
\begin{aligned}
E & \equiv\left(R_{\mu_{1} \mu_{2} \mu_{3} \mu_{4}}\right)^{2}-4\left(\mathcal{R}_{\mu_{1} \mu_{2}}\right)^{2}+\mathcal{R}^{2} \\
W & \equiv\left(C_{\mu_{1} \mu_{2} \mu_{3} \mu_{4}}\right)^{2}=\left(R_{\mu_{1} \mu_{2} \mu_{3} \mu_{4}}\right)^{2}-2\left(\mathcal{R}_{\mu_{1} \mu_{2}}\right)^{2}+\frac{1}{3} \mathcal{R}^{2},
\end{aligned}
$$

and $D^{2}$ is the square of the covariant derivative. The Riemann and Ricci tensors are:

$$
\begin{aligned}
R_{\mu_{2} \mu_{3} \mu_{4}}^{\mu_{1}} & \equiv \partial_{\mu_{3}} \Gamma_{\mu_{4} \mu_{2}}^{\mu_{1}}-\partial_{\mu_{4}} \Gamma_{\mu_{3} \mu_{2}}^{\mu_{1}}+\Gamma_{\mu_{3} \mu_{5}}^{\mu_{1}} \Gamma_{\mu_{4} \mu_{2}}^{\mu_{5}}-\Gamma_{\mu_{4} \mu_{5}}^{\mu_{1}} \Gamma_{\mu_{3} \mu_{2}}^{\mu_{5}}, \\
\mathcal{R}_{\mu_{2} \mu_{4}} & \equiv R_{\mu_{2} \mu_{1} \mu_{4}}^{\mu_{1}}, \quad \mathcal{R} \equiv \mathcal{R}_{\mu}^{\mu} .
\end{aligned}
$$

The LeviCivita connection is:

$$
\Gamma_{\mu_{2} \mu_{3}}^{\mu_{1}} \equiv \frac{1}{2} g^{\mu_{1} \mu_{4}}\left(\partial_{\mu_{2}} g_{\mu_{4} \mu_{3}}+\partial_{\mu_{3}} g_{\mu_{4} \mu_{2}}-\partial_{\mu_{4}} g_{\mu_{2} \mu_{3}}\right)
$$

and the metric has Euclidean signature. We also use the Minkowski signature $(+,--$ -) when discussing dispersion relations (Sections 2.1 and 4.2); on this metric, the trace anomaly (A.1) changes sign and the normalization $\lambda$ should be chosen negative.

For any local quantity $\mathcal{A}(x ; g)$ functional of $g^{\mu \mu^{\prime}}(x)$, we consider its $n$-th variation at the point $g^{\mu \mu^{\prime}}=\eta^{\mu \mu^{\prime}}$ and contract the pairs of indices with generic polarization tensors $h_{i}^{\mu \mu^{\prime}}$ as explained at the beginning of Section 2.3. We denote the $n$-th variation by:

$$
\mathcal{A}\left(x_{1}, h_{1} ; \ldots ; x_{n}, h_{n} ; x\right) \equiv h_{1}^{\mu_{1} \mu^{\prime}{ }_{1}} \frac{\delta}{\delta g^{\mu_{1} \mu^{\prime}}{ }_{1}\left(x_{1}\right)} \cdots h_{n}^{\mu_{n} \mu^{\prime}{ }_{n}} \frac{\delta}{\delta g^{\mu_{n} \mu^{\prime}{ }_{n}\left(x_{n}\right)}} \mathcal{A}(x ; g) .
$$

The differential of a functional $F[g]$ w.r.t. the metric, $g^{\mu \mu^{\prime}} \rightarrow g^{\mu \mu^{\prime}}+\lambda^{\mu \mu^{\prime}}$, with $\lambda$ an infinitesimal function, is:

$$
\delta_{\lambda} F[g]=F[g+\lambda]-F[g]=\int d x \frac{\delta F}{\delta g^{\mu \mu^{\prime}}(x)} \lambda^{\mu \mu^{\prime}}(x) .
$$

The Fourier transform of translation-invariant functions $f\left(x_{1}, x_{2}, . ., x_{n}\right)$ is defined by:

$$
f\left(x_{1}, x_{2}, . ., x_{n}\right)=(2 \pi)^{d-n d} \int d p_{1} \ldots d p_{n} \delta\left(\sum_{i=1}^{n} p_{i}\right) \exp \left(i \sum_{i=1}^{n} p_{i} \cdot x_{i}\right) f\left(p_{1}, \ldots \ldots, p_{n}\right) .
$$

In particular, the metric variation $(\mathrm{A.6})$ is translation invariant after flat-space limit and its Fourier transform is denoted by $\mathcal{A}\left(k_{1}, h_{1} ; \ldots ; k_{n}, h_{n}\right)$.

\section{B Diff and Weyl Ward identities}

The Diff Ward identities for the stress-tensor $n$-point functions are obtained by performing the reparametrization $\delta_{\epsilon}$ on the argument of the generating functional $W[g+h]$; here, $g^{\mu \nu}$ 
is the background metric and $h^{\mu \nu}$ is a small deformation, which is used as a source for extracting the stress-tensor correlators according to (2.19). The Diff transformations are:

$$
\begin{aligned}
& \delta_{\epsilon} g^{\mu \nu}=D^{(\mu} \epsilon^{\nu)} \\
& \delta_{\epsilon} h^{\mu \nu}=\left(D_{\sigma} \epsilon^{(\mu}\right) h^{\sigma \nu)}-(\epsilon \cdot D) h^{\mu \nu} .
\end{aligned}
$$

Upon expanding $W[g+h]$ in series of $h$ and performing the transformations, one finds that each term $O\left(h^{n}\right), n=0,1, \ldots$ yields the Ward identity for the $(n+1)$-point function (also involving the $n$-point correlator); the local form of the identity is obtained after integration by parts of the derivatives in (B.1, B.2) and the limit to flat space. To order $O\left(h^{0}\right)$, one finds $\partial_{\mu}\left\langle T_{\mu \nu}\right\rangle=0$, and to order $O(h)$ the two-point identity Eq.(2.23). The three-point identity (3.2) becomes simpler in momentum space and after contraction of indices with polarization tensors, as explained in Section 2.3.

The Weyl Ward identities are similarly obtained from Eq. (2.20), which we rewrite here:

$$
\delta_{\sigma} W[g] \equiv-2 \int \sqrt{g} g^{\mu \nu}\left\langle T_{\mu \nu}\right\rangle \sigma=-2 \int d x \sqrt{g} \mathcal{A}(x ; g) \sigma(x),
$$

with anomaly given by (A.1). Higher-point functions are obtained by differentiating both sides of this Equation w.r.t. to $g^{\mu \nu}\left(\int h \cdot \delta / \delta g\right)$ : on the left hand side, the variation can act on the explicit metric used for the trace or on v.e.v. yielding another stress-tensor; on the l.h.s., it can act on $\sqrt{g}$ or on the anomaly (whose variations are written in the notations (A.6, A.8)). After two variations, flat-space limit and Fourier transform, one finds Eq.(3.4), which we rewrite here:

$$
\begin{aligned}
& \left\langle\Theta\left(k_{3}\right)\left(T\left(k_{1}\right) \cdot h_{1}\right)\left(T\left(k_{2}\right) \cdot h_{2}\right)\right\rangle \\
& +\left[\left\langle\left(T\left(-k_{2}\right) \cdot h_{1}\right)\left(T\left(k_{2}\right) \cdot h_{2}\right)\right\rangle+\left\langle\left(T\left(k_{1}\right) \cdot h_{1}\right)\left(T\left(-k_{1}\right) \cdot h_{2}\right)\right\rangle\right] \\
& =\mathcal{A}\left(k_{1}, h_{1} ; k_{2}, h_{2}\right)-\frac{1}{2}\left[\left(h_{1}\right) \mathcal{A}\left(k_{2}, h_{2}\right)+\left(h_{2}\right) \mathcal{A}\left(k_{1}, h_{1}\right)\right] .
\end{aligned}
$$

As said in the text, the compatibility of this identity and its analogue for $\left(k_{3} \leftrightarrow k_{1}\right)$ imply the vanishing of the $\mathcal{R}^{2}$ term in the anomaly $(r=0)$, in agreement with the WessZumino consistency condition [12] [15]. The compatibility of the Diff and Weyl identities yields the equation:

$$
\begin{aligned}
0 & =\mathcal{A}\left(k_{1}, k_{1} \otimes v+v \otimes k_{1} ; k_{2}, h_{2}\right) \\
& +\mathcal{A}\left(k_{1}+k_{2}, v \otimes\left(h_{2} . k_{1}\right)+\left(h_{2} . k_{1}\right) \otimes v\right) \\
& -\left(k_{3} \cdot v\right) \mathcal{A}\left(k_{2}, h_{2}\right)-\left(k_{2} \cdot v\right) \mathcal{A}\left(k_{1}+k_{2}, h_{2}\right)-\left(k_{1} \cdot v\right) \mathcal{A}\left(k_{2}, h_{2}\right) \\
& -\frac{1}{2}\left(h_{2}\right) \mathcal{A}\left(k_{1}, v \otimes k_{1}+k_{1} \otimes v\right),
\end{aligned}
$$

which provides an useful check for the variations of the anomaly. The first variation of the Euler and Weyl terms vanishes in flat space and thus their second variation is transverse. 
The conformal isometries of the metric,

$$
0=\left(\delta_{\epsilon}+\delta_{\sigma}\right) g^{\mu \nu}(x) \equiv D^{(\mu} \epsilon^{\nu)}-2 \sigma(x) g^{\mu \nu}(x),
$$

imply Ward identities which are linear combinations of the previous Diff and Weyl identities. Let us consider the integrated expressions of the latter equations, say the $O\left(h^{n}\right)$ terms: these contain the $(n+1)$-point function multiplying $\delta_{\epsilon} g^{\mu \nu}$ and $\delta_{\sigma} g^{\mu \nu}$, respectively, plus the $n$-point functions and the $n$-th variation of the anomaly for the Weyl identity. By summing the two identities, the $(n+1)$-point functions cancel out for conformal isometries (B.6); after integration by parts of $\delta_{\epsilon} h$ in (B.2) plus $\delta_{\sigma} h=-2 \sigma h$, with vanishing surface term [29], the remaining $n$-point function contains the "conformal Lie derivative" $\left(\delta_{\epsilon}+\delta_{\sigma}\right) T\left(x_{i}\right)(3.23)$ acting on each stress tensor, $i=1, \ldots, n$; finally, there is the term with $n$-th variation of the trace anomaly. The result for $n=2$ is given by Eq.(3.22).

\section{Construction of the basic six-index tensors}

In this Section we construct the tensorial basis on which the correlators are decomposed. We start by considering the most general basis of linearly independent tensors with $2 n$ indices $(n=1,2,3), \mu_{1} \mu_{1}^{\prime} \cdots \mu_{n} \mu_{n}^{\prime}$, that are symmetric by exchange of any couple $\mu_{i} \leftrightarrow$ $\mu_{i}^{\prime}$. In addition we require those tensors to be built in terms of the available covariant quantities: $k_{1 \mu}, k_{2 \mu}, \eta_{\mu \mu^{\prime}}$. It is evident that all the symmetric 2 -tensors made of $k_{1}, k_{2}, \eta$ are generated by the following $N(2)=4$ 2-tensors:

$$
\left(k_{1} \cdot h_{1} \cdot k_{1}\right),\left(k_{2} \cdot h_{1} \cdot k_{2}\right),\left(k_{1} \cdot h_{1} \cdot k_{2}\right),\left(h_{1}\right)
$$

(indices are contracted with the polarization tensor $h_{1}$ as explained in Section 2.3).

The construction of symmetric 4 and 6 tensors made of $k_{1}, k_{2}, \eta$ is simplified by introducing the idea of primitive tensor: a primitive tensor is a tensor that is made by a connected chain of index contractions. For instance $\left(k_{1} \cdot h_{1} \cdot h_{2} \cdot k_{2}\right)$ is primitive, while $\left(k_{1} \cdot h_{1} \cdot h_{2} \cdot k_{2}\right)\left(h_{3}\right)$ is not. All the 2 -tensors of Eq.(C.1) are primitive.

Here follows the list of the $N_{p}(4)=5$ symmetric primitive 4-tensors:

$$
\left(k_{1} \cdot h_{2} \cdot h_{1} \cdot k_{1}\right),\left(k_{2} \cdot h_{2} \cdot h_{1} \cdot k_{2}\right),\left(k_{1} \cdot h_{2} \cdot h_{1} \cdot k_{2}\right),\left(k_{2} \cdot h_{2} \cdot h_{1} \cdot k_{1}\right),\left(h_{1} \cdot h_{2}\right),
$$

and of the $N_{p}(6)=13$ symmetric primitive 6-tensors,

$$
\begin{aligned}
& \left(k_{1} \cdot h_{3} \cdot h_{1} \cdot h_{2} \cdot k_{1}\right),\left(k_{1} \cdot h_{3} \cdot h_{2} \cdot h_{1} \cdot k_{1}\right),\left(k_{1} \cdot h_{2} \cdot h_{3} \cdot h_{1} \cdot k_{1}\right), \\
& \left(k_{2} \cdot h_{3} \cdot h_{1} \cdot h_{2} \cdot k_{2}\right),\left(k_{2} \cdot h_{3} \cdot h_{2} \cdot h_{1} \cdot k_{2}\right),\left(k_{2} \cdot h_{2} \cdot h_{3} \cdot h_{1} \cdot k_{2}\right), \\
& \left(k_{2} \cdot h_{3} \cdot h_{1} \cdot h_{2} \cdot k_{1}\right),\left(k_{2} \cdot h_{3} \cdot h_{2} \cdot h_{1} \cdot k_{1}\right),\left(k_{2} \cdot h_{2} \cdot h_{3} \cdot h_{1} \cdot k_{1}\right), \\
& \left(k_{1} \cdot h_{3} \cdot h_{1} \cdot h_{2} \cdot k_{2}\right),\left(k_{1} \cdot h_{3} \cdot h_{2} \cdot h_{1} \cdot k_{2}\right),\left(k_{1} \cdot h_{2} \cdot h_{3} \cdot h_{1} \cdot k_{2}\right), \\
& \left(h_{1} \cdot h_{2} \cdot h_{3}\right) .
\end{aligned}
$$


The complete basis of symmetric $2 n$-tensors can be constructed by multiplying in all possible ways primitive symmetric $2 k$ tensors $(1 \leq k \leq n)$ in such a way that the total number of free indices is $2 n$. In particular it is easily checked that the 4-tensors basis has dimension $N(4)=21=N_{p}(4)+N_{p}(2)^{2}$, while the 6-tensors are $137=N_{p}(6)+$ $3 N_{p}(4) N_{p}(2)+N_{p}(2)^{3}$.

For our purposes we also need the basis of 5 -tensors $E_{\mu_{1} \mu_{1}^{\prime} \mu_{2} \mu^{\prime}{ }_{2} \mu_{3}}$ made of $k_{1}, k_{2}, \eta$, that are symmetric by exchange of $\mu_{i} \leftrightarrow \mu_{i}^{\prime}$; they are contracted with $h_{1}, h_{2}$ and one polarization vector $v$. Again, the complete basis is found by multiplying primitive tensors in such a way to obtain 5 -tensors. In this case, we also need to consider the $N_{p}(1)=2$ primitive 1-tensors:

$$
\left(k_{1} \cdot v\right),\left(k_{2} \cdot v\right)
$$

the $N_{p}(3)=2$ primitive 3 -tensors:

$$
\left(k_{1} \cdot h_{1} \cdot v\right),\left(k_{2} \cdot h_{1} \cdot v\right)
$$

and the $N_{p}(5)=4$ primitive 5 -tensors,

$$
\left(v \cdot h_{2} \cdot h_{1} \cdot k_{1}\right),\left(k_{1} \cdot h_{2} \cdot h_{1} \cdot v\right),\left(v \cdot h_{2} \cdot h_{1} \cdot k_{2}\right),\left(k_{2} \cdot h_{2} \cdot h_{1} \cdot v\right) \text {. }
$$

We easily obtain $N(5)=62=N_{p}(5)+N_{p}(4) N_{p}(1)+2 N_{p}(3) N_{p}(2)+N_{p}(1) N_{p}(2)^{2} 5$-tensors.

Next, we need to impose the Bose symmetry realized by the exchange of $\left(k_{1}, h_{1}\right) \leftrightarrow$ $\left(k_{2}, h_{2}\right)$ on the 4 -tensors and 6 -tensors. This can be done by looking at the action on the primitive tensors: it is easy to realize then that the Bose symmetric 4-tensors are $N_{b}(4)=14$ and that the Bose symmetric 6-tensors are $N_{b}(6)=77$.

In Section 2.3, we defined the tensorial dimension $\delta_{T}$ of a tensor monomial as being the total number of $k_{1}, k_{2}$ with free indices (i.e. contracted with polarization tensors $h_{i}$ or $v)$. For instance $\delta_{T}((k 1 . h 2 . k 2)(k 2 . h 1 . k 2))=4$. The tensor dimension of a polynomial is the maximal tensorial dimension of the constituent monomials. Hereafter we report the dimensions of the tensors basis, divided according to the $\delta_{T}$ value, writing $\mathbf{m}_{\delta_{T}}$ for " $m$ tensors of tensorial dimension $\delta_{T} "$.

Classification of primitive tensors:

$$
\begin{aligned}
& N_{p}(1)=2=\mathbf{2}_{1}, \\
& N_{p}(2)=4=\mathbf{3}_{2}+\mathbf{1}_{0}, \\
& N_{p}(3)=2=\mathbf{2}_{1}, \\
& N_{p}(4)=5=\mathbf{4}_{2}+\mathbf{1}_{0}, \\
& N_{p}(5)=4=\mathbf{4}_{1}, \\
& N_{p}(6)=13=\mathbf{1 2}_{2}+\mathbf{1}_{0} .
\end{aligned}
$$


Classification of all tensors:

$$
\begin{aligned}
& N(2)=4=\mathbf{3}_{2}+\mathbf{1}_{0}, \\
& N(4)=21=\mathbf{9}_{4}+\mathbf{1 0}_{2}+\mathbf{2}_{0}, \\
& N(5)=62=\mathbf{1 8}_{5}+\mathbf{3 2}_{3}+\mathbf{1 2}_{1}, \\
& N(6)=137=\mathbf{2 7}_{6}+\mathbf{6 3}_{4}+\mathbf{4 2}_{2}+\mathbf{5}_{0} .
\end{aligned}
$$

Classification of all Bose-symmetric tensors:

$$
\begin{aligned}
& N_{b}(4)=14=\mathbf{6}_{4}+\mathbf{6}_{2}+\mathbf{2}_{0} \\
& N_{b}(6)=77=\mathbf{1 5}_{6}+\mathbf{3 4}_{4}+\mathbf{2 4}_{2}+\mathbf{4}_{0} .
\end{aligned}
$$

\section{Long formulae}

Here we report the explicit form of the "null vector" which occurs in the $(1 \leftrightarrow 2)$ symmetric six-index tensors basis $\mathcal{P}_{i}, i=1 \ldots, 77$, (Table 1) in four dimensions; this vector expresses the linear dependence in this basis and should be put to zero (Section 3 ). The null vector is $\mathcal{T}_{8}$ in Eq.(3.8); it reads:

$$
\begin{aligned}
\mathcal{T}_{8} & =2\left(\mathcal{P}_{17}+\mathcal{P}_{20}-\mathcal{P}_{26}-\mathcal{P}_{27}+\mathcal{P}_{29}-\mathcal{P}_{33}-\mathcal{P}_{34}+\mathcal{P}_{46}+\mathcal{P}_{49}\right)-\mathcal{P}_{36}-\mathcal{P}_{39}-\mathcal{P}_{41}-\mathcal{P}_{43} \\
& +2 k^{2}\left(\mathcal{P}_{50}+\mathcal{P}_{51}+\mathcal{P}_{52}-\mathcal{P}_{60}-\mathcal{P}_{61}-\mathcal{P}_{66}\right) \\
& +\left(2 k^{2}-q^{2}\right)\left(\mathcal{P}_{53}+\mathcal{P}_{54}+\mathcal{P}_{55}+\mathcal{P}_{56}+\mathcal{P}_{71}+\mathcal{P}_{73}-\mathcal{P}_{59}-\mathcal{P}_{62}-\mathcal{P}_{63}-\mathcal{P}_{65}-\mathcal{P}_{67}-\mathcal{P}_{68}\right) \\
& +k^{2}\left(-\mathcal{P}_{57}-\mathcal{P}_{58}-\mathcal{P}_{64}+\mathcal{P}_{69}+\mathcal{P}_{70}+\mathcal{P}_{72}\right) \\
& +\frac{q^{2}}{4}\left(4 k^{2}-q^{2}\right)\left(-2 \mathcal{P}_{74}+\mathcal{P}_{75}+\mathcal{P}_{76}-\mathcal{P}_{77}\right)=0
\end{aligned}
$$

Note that this tensor has $\delta_{T}=4$, because it possesses no components in the first 15 elements of the basis; moreover, it is easy to check that its scale dimension is $\Delta=4$

Next, we write the complete set of reduction formulae (Section 4.1) which determine the $13\langle T T T\rangle$ amplitudes $A_{i}\left(q^{2}, k^{2}=0\right)$ of the $\delta_{T}=6$ tensors (see Table 3): for $i=$ $1, \ldots, 7$, they correspond to the traceless transverse tensors, for $i=9,10$, to the anomalies $\left(A_{E}, A_{W}\right)$, and for $i=15,16,17$, to the traceful transverse tensor that vanish at the Weylinvariant critical points. The formulae express the amplitudes in terms of the coefficients $u_{i}$ of the $\delta_{T}=6$ tensors $\mathcal{P}_{i}, i=1, \ldots, 15$.

Three amplitudes and the two constraints among the $u_{i}$ 's were already given in the text, see Eqs.(4.24.5); the remaining $7+3$ amplitudes are:

$$
A_{1}=u_{14}, \quad A_{2}=u_{2}, \quad A_{4}=u_{4},
$$




$$
\begin{aligned}
& A_{3}=\frac{1}{36}\left(4 u_{2}-27 u_{3}-4 u_{4}-u_{5}+9 u_{7}+11 u_{8}-2 u_{11}+12 u_{14}+3 u_{15}\right), \\
& A_{5}=\frac{1}{72}\left(-28 u_{2}+81 u_{3}+28 u_{4}+7 u_{5}-14 u_{8}+5 u_{11}-12 u_{14}-3 u_{15}\right), \\
& A_{6}=\frac{1}{288}\left(-40 u_{2}+99 u_{3}+40 u_{4}+10 u_{5}-20 u_{8}-18 u_{9}+20 u_{11}-12 u_{14}-3 u_{15}\right), \\
& A_{7}=u_{7},
\end{aligned}
$$

and

$$
\begin{aligned}
& A_{15}=\frac{1}{3}\left(4 u_{2}-4 u_{4}-u_{5}+2 u_{8}+12 u_{9}+3 u_{10}-2 u_{11}\right) \\
& A_{16}=\frac{1}{3}\left(4 u_{2}-4 u_{4}-u_{5}+2 u_{8}+u_{11}+3 u_{13}\right) \\
& A_{17}=\frac{1}{27}\left(8 u_{2}-27 u_{3}-8 u_{4}-2 u_{5}+27 u_{6}+4 u_{8}-4 u_{11}-12 u_{14}-3 u_{15}\right) .
\end{aligned}
$$

The corresponding formulae for non-vanishing $k^{2}$ can be found in the Mathematica notebook. At criticality, there are 3 further constraints (4.8) among the $u_{i}$ 's, which are equivalent to the conditions $A_{i}=0$, for $i=15,16,17$.

\section{References}

[1] A. B. Zamolodchikov, JETP Lett. 43 (1986) 730; Sov. J. Nucl. Phys. 46 (1987) 1090.

[2] A. Cappelli, G. D'Appollonio, R. Guida and N. Magnoli, On the c-theorem in more than two dimensions, Int. Conf. "Non-perturbative Quantum Effects 2000", Paris, Sept. 2000, ed. D. Bernard and B. Julia, JHEP proceedings (2000).

[3] J. L. Cardy, Phys. Lett. B215 (1988) 749.

[4] I. Jack and H. Osborn, Nucl. Phys. B343 (1990) 647.

[5] A. Cappelli, D. Friedan, J. I. Latorre, Nucl. Phys. B352 (1991) 616.

[6] F. Bastianelli, Phys. Lett. B369 (1996) 249; D. Anselmi, D. Z. Freedman, M.T. Grisaru and A.A. Johansen, Nucl. Phys. B526 (1998) 543.

[7] L. Girardello, M. Petrini, M. Porrati and A. Zaffaroni, JHEP 9812 (1998) 022; D. Z. Freedman, S. S. Gubser, K. Pilch and N. P. Warner, Adv. Theor. Math. Phys. 3 (1999) 363; for a review, see: S. S. Gubser, Class. Quantum Grav. 17 (2000) 1081, D. Z. Freedman and P. Henry-Labordère, hep-th/00011086.

[8] D. Anselmi, Phys. Lett. B476 (2000) 182; D. Anselmi, L. Girardello, M. Porrati and A. Zaffaroni, Phys. Lett. B481 (2000) 346; M. Porrati and A. Starinets, preprint hep-th/0009198 and Phys. Lett. B498 (2001) 285. 
[9] S. Forte and J. I. Latorre, Nucl. Phys. B535 (1998) 709.

[10] D. Anselmi, Ann. Phys. 276 (1999) 361; Nucl. Phys. B567 (2000) 331.

[11] A. Cappelli and G. D'Appollonio, Phys. Lett. B487 (2000) 87.

[12] For a review, see: Current algebra and anomalies, Treiman, R. Jackiw, B. Zumino and E. Witten eds, World Scientific (1985), Singapore.

[13] For a review, see: M. J. Duff, Class. Quantum Grav. 11 (1984) 1387.

[14] P. Pasti, M. Bregola and L. Bonora, Class. Quantum Grav. 3 (1986) 635.

[15] A. Cappelli and A. Coste, Nucl. Phys. B314 (1989) 707.

[16] S. Deser and A. Schwimmer, Phys. Lett. B309 (1993) 279 .

[17] P. Di Francesco, P. Mathieu and D. Senechal, Conformal Field Theories, SpringerVerlag (1996), Berlin.

[18] J. L. Cardy, Phys. Rev. Lett. 60 (1988) 2709.

[19] Y. Stanev, Bulgarian J. Phys. 15 (1988) 2; H. Osborn and A. C. Petkou, Ann. Phys. 231 (1994) 311; J. Erdmenger and H. Osborn, Nucl. Phys. B483 (1997) 431.

[20] H. Osborn and J. I. Latorre, Nucl. Phys. B511 (1998) 737.

[21] H. Osborn and G. Shore, Nucl. Phys. B571 (2000) 287.

[22] A. D. Dolgov and V. I. Zakharov, Nucl. Phys. B27 (1971) 525; Y. Frishman, A. Schwimmer, T. Banks and S. Yankielowicz, Nucl. Phys. B177 (1981) 157.

[23] For a review see: N. D. Birrel and P .C. W. Davies, Quantum Fields in Curved Space, Cambridge Univ. Press (1982), Cambridge.

[24] A. Cappelli, J. I. Latorre and X. Vilasis-Cardona, Nucl. Phys. B376 (1992) 510.

[25] J. Horejsi and O. Teryaev, Z. Phys. C 65 (1995) 691.

[26] S. Wolfram, Mathematica, Cambridge Univ. Press (1996), Cambridge.

[27] L. Rosemberg, Phys. Rev. 129 (1963) 2786.

[28] J. Polchinski, Nucl. Phys. B303 (1988) 226.

[29] J. L. Cardy, in Phase transitions and critical phenomena, C. Domb and L. Lebowitz eds, vol. 11, (Academic Press, New York, 1988).

[30] S. Coleman and B. Grossman, Nucl. Phys. B203 (1982) 205. 